\title{
1 A flexible observed factor model with separate dynamics for the factor volatilities and their correlation matrix
}

\author{
Yu-Cheng $\mathrm{Ku}^{a, b *}$, Peter Bloomfield ${ }^{a}$, Robert $\mathrm{Kohn}^{b}$ \\ ${ }^{a}$ Department of Statistics, North Carolina State University, Raleigh, NC 27695, USA \\ ${ }^{b}$ School of Economics, University of New South Wales, Kensington, NSW 2052, Australia
}

October 30, 2018

\begin{abstract}
Our article considers a regression model with observed factors. The observed factors have a flexible stochastic volatility structure that has separate dynamics for the volatilities and the correlation matrix. The correlation matrix of the factors is time-varying and its evolution is described by an inverse Wishart process. The model specifies the evolution of the observed volatilities flexibly and is particularly attractive when the dimension of the observations is high. A Markov chain Monte Carlo algorithm is developed to estimate the model. It is straightforward to use this algorithm to obtain the predictive distributions of future observations and to carry out model selection. The model is illustrated and compared to other Wishart-type factor multivariate stochastic volatility models using various empirical data including monthly stock returns and portfolio weighted returns. The evidence suggests that our model has better predictive performance. The paper also allows the idiosyncratic errors to follow individual stochastic volatility processes in order to deal with more volatile data such as daily or weekly stock returns.
\end{abstract}

Keywords: Correlated factors; Inverse Wishart; Markov chain Monte Carlo.

\footnotetext{
${ }^{*}$ Corresponding author. Tel: +61-04-32561617; fax: +61-04-93136337. E-mail: yku2@ncsu.edu
} 


\section{Introduction}

For the last two decades, multivariate stochastic volatility (MSV) models have been an important class of models in financial econometrics. Recent developments in this area focus on dimension reduction since the complexity of computation and the difficulty in model interpretation grow drastically as the dimension of the model increases. Harvey et al. (1994) were the first to discuss a factor structure for MSV models. The seminal work by Jacquier et al. (1995) introduced Bayesian approaches to the factor MSV (FMSV) literature. The FMSV model is also considered and discussed by Pitt and Shephard (1999), Chib et al. (2006), Lopes and Carvalho (2007), among others. A common feature in these FMSV models is that they impose the diagonality assumption on the factor correlation or covariance matrices, implying that the factors are uncorrelated. However, it is often unrealistic to assume that the factors do not interact with each other, especially when the factors are observed.

To relax the diagonality assumption, Philipov and Glickman (2006b) introduces a timevarying FMSV model in which the inverse factor covariance matrices are driven by Wishart processes. The model is a direct application of Philipov and Glickman (2006a) to the factor structure. The inverse Wishart specification introduced by Philipov and Glickman (2006a,b) has the attractive property that it can be easily incorporated into model estimation with Bayesian Markov chain Monte Carlo (MCMC) methods. Based on a similar setting, Asai and McAleer (2009) also proposes an MSV model, where the individual return series is modeled with the stochastic volatility (SV) process and the covariance process is characterized by the inverse Wishart distribution. Asai and McAleer (2009) call this type of model a "Wishart Inverse Covariance" (WIC) model.

When the vector of dependent variables is high-dimensional, the WIC models proposed by Philipov and Glickman (2006a) and Asai and McAleer (2009) have two problems. First, the computation becomes highly time-consuming as the dimension increases. Second, the time effect among the different series is controlled by just one scalar persistence parameter, which is likely to 
be too restrictive in real applications. The factor structure proposed in Philipov and Glickman (2006b) helps resolve the first problem. However, when it comes to more factors, say, three or more, these distinct underlying factors still have to share a common time effect controlled by a single persistence parameter; hence, the second problem remains. In order to solve the two problems simultaneously, we propose in our article an observed dynamic-correlation FMSV model (O-DCFMSV).

The basic model form of O-DCFMSV is similar to that of Asai and McAleer (2009), but the structure is applied to a factor model. Consequently, compared to Asai and McAleer (2009), the O-DCFMSV model has advantages in both model estimation and in interpretation for high-dimensional data. Moreover, since in the O-DCFMSV model we allow different time effects on the factors through separate SV processes, it is more flexible compared to the Philipov and Glickman (2006b). To estimate the model, we develop an MCMC algorithm that deals with all unknown parameters and latent variables jointly, which is quite different from the partial MCMC approach used in Asai and McAleer (2009). Using our approach, prediction and model selection become straightforward, issues that are not dealt with in either Philipov and Glickman (2006b) or Asai and McAleer (2009). We illustrate how to implement the one-step-ahead prediction, by which we can forecast many quantities of interest, such as the return series, the return covariance matrix, the correlation matrix of the factors, and the value at risk (VaR) of a portfolio. We can also conduct model selection based on predictive performance.

To summarize, the contribution of our paper is twofold. First, it introduces a flexible factor model to the MSV literature. Secondly, the MCMC algorithm designed in this paper can be used for prediction and model selection, which significantly extends the usefulness of the WIC models in real problems.

The remainder of the paper is organized as follows. Section 2 presents the model and the MCMC algorithm to estimate the model. Section 3 conducts a simulation study to illustrate the model. Section 4 provides two empirical examples. The O-DCFMSV model is applied to 
1 portfolio and stock return data and is compared to the model of Philipov and Glickman (2006b)

2 based on the quality of one-step-ahead predictions. Section 5 extends the model to the case

3 where the idiosyncratic error terms are allowed to follow independent SV processes. Section 6 4 concludes the paper.

\section{The Model}

\section{$6 \quad 2.1$ Model Specification}

7 Suppose that at time $t$ we have $p$ asset returns, $\boldsymbol{y}_{t}$, and $q$ underlying observed factors, $\boldsymbol{f}_{t}$, such 8 that

$$
\boldsymbol{y}_{t}=\boldsymbol{B} \boldsymbol{f}_{t}+\boldsymbol{e}_{t}
$$

where the $\left\{\boldsymbol{f}_{t}, t \geq 1\right\}$ and $\left\{\boldsymbol{e}_{t}, t \geq 1\right\}$ are independent stochastic processes. The $\boldsymbol{e}_{t}$ are also assumed to be independent with $\boldsymbol{e}_{t} \sim \mathrm{N}_{p}\left(\boldsymbol{e}_{t} \mid \mathbf{0}, \boldsymbol{\Omega}\right), \boldsymbol{\Omega}=\operatorname{diag}\left(\sigma_{1}^{2}, \ldots, \sigma_{p}^{2}\right)$, where $\mathrm{N}_{p}(\boldsymbol{X} \mid \boldsymbol{\mu}, \boldsymbol{\Sigma})$ is a $p$-dimensional multivariate normal density in $\boldsymbol{X}$ with mean $\boldsymbol{\mu}$ and covariance matrix $\boldsymbol{\Sigma}$. The assumption that the conditional variance of $\boldsymbol{e}_{t}$ is constant is relaxed in Section 5 to allow $\boldsymbol{e}_{t}$ to have SV dynamics. The model for the factors is as follows:

$$
\begin{aligned}
\boldsymbol{f}_{t} & =\boldsymbol{V}_{t}^{1 / 2} \boldsymbol{\epsilon}_{t}, \\
\boldsymbol{V}_{t}^{1 / 2} & =\operatorname{diag}\left(e^{h_{t 1} / 2}, e^{h_{t 2} / 2}, \ldots, e^{h_{t q} / 2}\right), q \leq p, \\
\boldsymbol{h}_{t+1} & =\boldsymbol{\mu}+\boldsymbol{\phi} \circ\left(\boldsymbol{h}_{t}-\boldsymbol{\mu}\right)+\boldsymbol{\eta}_{t}, \\
h_{1 i} & \sim \mathrm{N}\left(h_{1 i} \mid \mu_{i}, \frac{\sigma_{\eta, i}^{2}}{1-\phi_{i}^{2}}\right), i=1,2, \ldots, q,
\end{aligned}
$$

where $\mathrm{N}\left(x \mid \mu, \sigma^{2}\right)$ is a univariate normal distribution in $x$ with mean $\mu$ and $\sigma^{2}$, and $\circ$ is the elementwise multiplication operator. The stochastic sequences $\left\{\boldsymbol{\epsilon}_{t}, t \geq 1\right\}$ and $\left\{\boldsymbol{\eta}_{t}, t \geq 1\right\}$ are 
independent with $\boldsymbol{\eta}_{t}$ also an independent sequence and

$$
\begin{aligned}
\boldsymbol{\epsilon}_{t} \mid \boldsymbol{P}_{t} & \sim \mathrm{N}_{q}\left(\boldsymbol{\epsilon}_{t} \mid 0, \boldsymbol{\Sigma}_{\epsilon, t}\right), \\
\boldsymbol{\eta}_{t} & \sim \mathrm{N}_{q}\left(\boldsymbol{\eta}_{t} \mid 0, \boldsymbol{\Sigma}_{\eta}\right), \quad \boldsymbol{\Sigma}_{\eta}=\operatorname{diag}\left(\sigma_{\eta, 1}^{2}, \ldots, \sigma_{\eta, q}^{2}\right) .
\end{aligned}
$$

The covariance matrix $\boldsymbol{\Sigma}_{\epsilon, t}$ is a correlation matrix which is obtained by standardizing the $q \times q$ stochastic covariance matrix $\boldsymbol{P}_{t}$ so that

$$
\boldsymbol{\Sigma}_{\epsilon, t}=\left(\operatorname{diag} \boldsymbol{P}_{t}\right)^{-\frac{1}{2}} \boldsymbol{P}_{t}\left(\operatorname{diag} \boldsymbol{P}_{t}\right)^{-\frac{1}{2}}
$$

The dynamics of $\boldsymbol{P}_{t}$, and hence $\boldsymbol{\Sigma}_{\epsilon, t}$ are given by the stationary autoregressive inverse Wishart process

$$
\boldsymbol{P}_{t+1}^{-1} \mid k, \boldsymbol{P}_{t}^{-1} \sim \mathrm{W}_{q}\left(\boldsymbol{P}_{t+1}^{-1} \mid k, \boldsymbol{S}_{t}\right), \quad \boldsymbol{S}_{t}=\frac{1}{k} \boldsymbol{P}_{t}^{-\frac{d}{2}} \boldsymbol{A} \boldsymbol{P}_{t}^{-\frac{d}{2}},
$$

where $\mathrm{W}_{q}(\boldsymbol{X} \mid k, \boldsymbol{S})$ is a $q \times q$ Wishart density in $\boldsymbol{X}$ with degrees of freedom (df) $k \geq q$ and the scale matrix $\boldsymbol{S}$. The $q \times q$ matrix $\boldsymbol{A}$ is a symmetric positive definite matrix parameter, and $d$ is a scalar parameter that accounts for the memory of the matrix process $\left\{\boldsymbol{P}_{t}\right\}$. The matrix power operation $\boldsymbol{P}_{+}^{-d / 2}$ is defined by a spectral decomposition. Similarly to Philipov and Glickman (2006a,b) and Asai and McAleer (2009), we set the initial value $\boldsymbol{P}_{0}$ to be $\boldsymbol{P}_{0}=\boldsymbol{I}_{q}$ for convenience.

In the WIC context, there are two different ways to define the scale matrix. Asai and McAleer (2009) uses the specification (5), while Philipov and Glickman (2006b) uses a BEKK-type representation

$$
\boldsymbol{S}_{t-1}=\frac{1}{k} \boldsymbol{A}^{\frac{1}{2}}\left(\boldsymbol{P}_{t-1}^{-1}\right)^{d}\left(\boldsymbol{A}^{\frac{1}{2}}\right)^{\prime}
$$

where $A^{\frac{1}{2}}$ is defined by a Cholesky decomposition such that $\boldsymbol{A}=\boldsymbol{A}^{\frac{1}{2}}\left(\boldsymbol{A}^{\frac{1}{2}}\right)^{\prime}$. In either case, Philipov and Glickman (2006a) and Asai and McAleer (2009) show that $\log \left|\boldsymbol{P}_{t+1}\right|$ is a first-order 
There are two set of parameters in the measurement equation (11). For $\boldsymbol{\Omega}=\operatorname{diag}\left(\sigma_{1}^{2}, \ldots, \sigma_{p}^{2}\right)$, following Liesenfeld and Richard (2006), we assign independent inverse gamma priors for the idiosyncratic variances $\sigma_{j}^{2}$. Specifically, $\sigma_{j}^{2} \sim \operatorname{IG}\left(\sigma_{j}^{2} \mid\right.$ shape $=\nu_{0} / 2$, scale $\left.=\nu_{0} s_{0} / 2\right), j=1, \ldots, p$. In all our analyses, we use $\nu_{0}=10$ and $s_{0}=0.01$. This defines a vague prior which is commonly adopted in the literature. For the loading matrix $\boldsymbol{B}$, following Jacquier et al. (1995), we choose

the prior given by

$$
p(\boldsymbol{B} \mid \boldsymbol{\Omega}) \propto|\boldsymbol{\Omega}|^{-p / 2} \operatorname{etr}\left(-\frac{1}{2} \boldsymbol{\Omega}^{-1} \boldsymbol{B} \boldsymbol{B}^{\prime}\right),
$$

autoregression with autoregressive parameter $d$; if $d \in(-1,1)$, then this first order autoregressive process is stationary. We have also conducted simulations that suggest that the whole process $\boldsymbol{P}_{t}$ is stationary for $d \in(-1,1)$.

Although the O-DCFMSV model has some similarities with Asai and McAleer (2009) in model specification, the two models are in fact different in several respects. First, Asai and McAleer (2009) adopt the settings (2a) - (5) to model the return series, while in O-DCFMSV, we apply the settings to the observed factors. As a matter of fact, this is the main advantage of O-DCFMSV. When it comes to a high-dimensional environment, the estimation of the model of Asai and McAleer (2009) is extremely tedious. The reason is that the model itself is defined through a sequence of spectral decompositions or singular value decompositions, and the posterior densities of the parameters are complicated and dependent on the data dimension. The second difference is in the sampling scheme to estimate the model which we discuss below.

\subsection{Priors}

where $\operatorname{etr}(\boldsymbol{X})$ means $\exp (\operatorname{trace}(\boldsymbol{X}))$. This prior implies that the columns $\boldsymbol{B}_{i}$ of $\boldsymbol{B}$ are a priori independent, each with a prior $\mathrm{N}_{p}\left(\boldsymbol{B}_{i} \mid \mathbf{0}, \boldsymbol{\Omega}\right)$, which is uninformative relative to the data.

The priors for the SV parameters are as follows. We adopt the default settings by Kim et al. (1998). For the mean $\mu_{i}$ and variance $\sigma_{\eta, i}^{2}, i=1, . ., q$, we respectively assume that $\mu_{i} \sim$ 
$1 \mathrm{~N}\left(\mu_{i} \mid 0,10\right)$ and $\sigma_{\eta, i}^{2} \sim \operatorname{IG}\left(\sigma_{\eta, i}^{2} \mid 5,0.05\right)$. The prior for $\phi_{i}$ is a shifted and scaled beta distribution.

2 Let $\phi_{i}=2 \tilde{\phi}_{i}-1$ where $\tilde{\phi}_{i} \sim \operatorname{Beta}\left(\phi^{(1)}, \phi^{(2)}\right)$. We choose $\phi^{(1)}=20$ and $\phi^{(2)}=1.5$, implying a 3 prior mean of $2 \phi^{(1)} /\left(\phi^{(1)}+\phi^{(2)}\right)-1=0.86$.

$4 \quad$ The priors for the correlation-level parameters are chosen as follow: for $\boldsymbol{A}$ we specify the prior $\boldsymbol{A}^{-1} \sim \mathrm{W}_{q}\left(\boldsymbol{A}^{-1} \mid q, q^{-1} \boldsymbol{I}_{q}\right)$, which implies a prior mean of $\boldsymbol{I}_{q}$; for $d$, we choose the vague 6rior $d \sim \operatorname{Unif}(d \mid-1,1)$. Finally, for $k$ we set $k \sim \lambda_{0} e^{-\lambda_{0}} I_{(q, \infty)}(k)$. Note that the prior for $7 \quad k$ is a truncated exponential distribution with a rate parameter $\lambda_{0}$. Throughout the paper we 8 set $\lambda_{0}=0.02$. This implies a prior mean of $50+q$ and a prior standard deviation of 50 , which specifies a diffuse prior.

\subsection{MCMC Estimation}

\subsubsection{Joint Distribution}

We estimate the model using the MCMC simulation method described below. Let the observed data $\boldsymbol{Y}=\left\{\boldsymbol{y}_{t}\right\}: T \times p, \boldsymbol{F}=\left\{\boldsymbol{f}_{t}\right\}: T \times q$, the log volatilities $\boldsymbol{H}=\left\{\boldsymbol{h}_{t}\right\}: T \times q$, the normalized factors $\boldsymbol{\epsilon}=\left\{\boldsymbol{\epsilon}_{t}\right\}: T \times p$, and the sequence of unnormalized covariance matrices $\boldsymbol{P}=\left\{\boldsymbol{P}_{t}, t=\right.$ $1, \ldots, T\}$. Let $\boldsymbol{\omega}=\left\{\boldsymbol{\omega}_{i}, i=1, \ldots q\right\}$, with $\boldsymbol{\omega}_{i}=\left\{\boldsymbol{\mu}_{i}, \boldsymbol{\phi}_{i}, \boldsymbol{\sigma}_{\eta, i}, i=1, \ldots, q\right\}$, be the parameters of the volatilities of the factors.

The joint density of $(\boldsymbol{Y}, \boldsymbol{F}, \boldsymbol{H}, \boldsymbol{\epsilon}, \boldsymbol{P}, \boldsymbol{B}, \boldsymbol{\Omega}, \omega, \boldsymbol{A}, d, k)$ is

$$
\begin{aligned}
p(\boldsymbol{Y}, \boldsymbol{F}, \boldsymbol{H}, \boldsymbol{\epsilon}, \boldsymbol{P}, \boldsymbol{B}, \boldsymbol{\Omega}, \boldsymbol{\omega}, A, d, k) & =p(\boldsymbol{Y} \mid \boldsymbol{B}, \boldsymbol{F}, \boldsymbol{\Omega}) p(\boldsymbol{F} \mid \boldsymbol{H}, \boldsymbol{\epsilon}) p(\boldsymbol{H} \mid \boldsymbol{\omega}) p(\boldsymbol{\epsilon} \mid \boldsymbol{P}) \\
& \times p(\boldsymbol{P} \mid \boldsymbol{A}, d, k) p(\boldsymbol{B} \mid \boldsymbol{\Omega}) p(\boldsymbol{\Omega}) p(\boldsymbol{A}) p(d) p(k),
\end{aligned}
$$


where

$$
\begin{aligned}
p(\boldsymbol{Y} \mid \boldsymbol{B}, \boldsymbol{F}, \boldsymbol{\Omega}) & =\prod_{t=1}^{T} p\left(\boldsymbol{y}_{t} \mid \boldsymbol{f}_{t}, \boldsymbol{\Omega}\right), \\
p(\boldsymbol{F} \mid \boldsymbol{H}, \boldsymbol{\epsilon}) & =\prod_{t=1}^{T} p\left(\boldsymbol{f}_{t} \mid \boldsymbol{h}_{t}, \boldsymbol{\epsilon}_{t}\right), \\
p\left(\boldsymbol{h}_{t} \mid \boldsymbol{\omega}\right) & =p\left(\boldsymbol{h}_{1} \mid \boldsymbol{\omega}\right) \prod_{t=2}^{T} p\left(\boldsymbol{h}_{t} \mid \boldsymbol{h}_{t-1}, \boldsymbol{\omega}\right), \\
p\left(\boldsymbol{h}_{1} \mid \boldsymbol{\omega}\right) & =\prod_{i=1}^{q} p\left(h_{1 i} \mid \boldsymbol{\omega}_{i}\right), \quad p\left(\boldsymbol{h}_{t} \mid \boldsymbol{h}_{t-1}, \boldsymbol{\omega}\right)=\prod_{i=1}^{q} p\left(\boldsymbol{h}_{t i} \mid \boldsymbol{h}_{t-1, i}, \boldsymbol{\omega}_{i}\right), \\
p(\boldsymbol{\epsilon} \mid \boldsymbol{P}, \boldsymbol{A}) & =\prod_{t=1}^{T} p\left(\boldsymbol{\epsilon}_{t} \mid \boldsymbol{P}_{t}\right), \\
p(\boldsymbol{P} \mid \boldsymbol{A}, d, k) & =\prod_{t=1}^{T} p\left(\boldsymbol{P}_{t} \mid \boldsymbol{P}_{t-1}, \boldsymbol{A}, d, k\right) .
\end{aligned}
$$

1 The densities $p\left(\boldsymbol{y}_{t} \mid \boldsymbol{f}_{t}, \boldsymbol{\Omega}\right)$ in (9a) are given by Eq. (1). The densities $p\left(\boldsymbol{f}_{t} \mid \boldsymbol{h}_{t}, \boldsymbol{\epsilon}_{t}\right)$ in (9b) are 2 degenerate and are given by (2a). The densities $p\left(h_{1 i} \mid \boldsymbol{\omega}_{i}\right)$ in (9c) are given by (2d) , and the densities $p\left(\boldsymbol{h}_{t i} \mid \boldsymbol{h}_{t-1, i}, \boldsymbol{\omega}_{i}\right)$ in (9c) are given by (2c). The densities $p\left(\boldsymbol{\epsilon}_{t} \mid \boldsymbol{P}_{t}\right)$ in (9d) are given by

4 (3a) and (4). The densities $p\left(\boldsymbol{P}_{t} \mid \boldsymbol{P}_{t-1}, \boldsymbol{A}, d, k\right)$ in (9e) are given by (5). The priors $p(\boldsymbol{B} \mid \boldsymbol{\Omega})$, $5 \quad p(\boldsymbol{\Omega}), p(\boldsymbol{A}), p(d)$, and $p(k)$ are discussed in the previous section.

6

7 We sample from the following conditional distributions. For $\sigma_{j}^{2}$, we sample from the inverse 8

10

\subsubsection{Conditional Distributions} gamma distribution:

$$
\begin{aligned}
p\left(\sigma_{j}^{2} \mid \text { rest }\right) & \propto p\left(\sigma_{j}^{2}\right) \cdot p\left(\boldsymbol{y}_{j} \mid \sigma_{j}^{2}, \boldsymbol{B}, \boldsymbol{F}\right) \\
& \propto\left(\sigma_{j}^{2}\right)^{-\frac{\nu_{0}+T}{2}-1} \exp \left\{-\frac{1}{2 \sigma_{j}^{2}}\left[\nu_{0 j} s_{0 j}+\sum_{t=1}^{T}\left(y_{t j}-\sum_{i=1}^{q} b_{j i} f_{t i}\right)^{2}\right]\right\},
\end{aligned}
$$

9 where $y_{t j}$ is the $j$ th element of $\boldsymbol{y}_{t}, f_{t i}$ is the $i$ th element of $\boldsymbol{f}_{t}$, and $b_{j i}$ denotes the $i j$ th element of $\boldsymbol{B}$. It follows from (10) that the conditional density of $\sigma_{j}^{2}$ is an inverse gamma with the shape 
1 parameter $\frac{\nu_{0}+T}{2}$ and the scale parameter $\frac{1}{2}\left[\nu_{0 j} s_{0 j}+\sum_{t=1}^{T}\left(y_{t j}-\sum_{i=1}^{q} b_{j i} f_{t i}\right)^{2}\right]$.

The posterior density of $\boldsymbol{B}$ is a matrix variate normal density given by:

$$
\begin{aligned}
p(\boldsymbol{B} \mid \text { rest }) & \propto p(\boldsymbol{B} \mid \boldsymbol{\Omega}) \cdot p(\boldsymbol{Y} \mid \boldsymbol{B}, \boldsymbol{\Omega}, \boldsymbol{F}) \\
& \propto \operatorname{etr}\left(-\frac{1}{2}\left\{\boldsymbol{\Omega}^{-1}\left[\left(\boldsymbol{B}-\boldsymbol{\mu}_{B}\right) \boldsymbol{\Sigma}_{B}^{-1}\left(\boldsymbol{B}-\boldsymbol{\mu}_{B}\right)^{\prime}\right]\right\}\right),
\end{aligned}
$$

3 where $\boldsymbol{\Sigma}_{B}=\left(\boldsymbol{F}^{\prime} \boldsymbol{F}+\boldsymbol{I}\right)^{-1}$ and $\boldsymbol{\mu}_{B}=\boldsymbol{Y}^{\prime} \boldsymbol{F} \boldsymbol{\Sigma}_{B}$.

We now follow Kim et al. (1998) and discuss how to sample the SV parameters $\boldsymbol{\omega}$ and $\boldsymbol{H}$. First we transform the SV equation (2b) into a linear model by taking the logarithm:

$$
f_{t i}^{*}=h_{t i}+z_{t i}
$$

where $f_{t i}^{*}=\log \left(f_{t i}^{2}+c\right)$ and $z_{t i}$ is a $\log \chi_{1}^{2}$ random variable. The scalar $c$ is an "offset" constant that is set to be $10^{-5}$. Following Kim et al. (1998), the distribution of $f_{t i}^{*}$ can be approximated by a seven-component normal mixture with the component indicator variables $s=\left\{s_{t i}\right\}$. Using the offset mixture integration sampler developed by Kim et al. (1998), for each $i=1, . ., q$ we sample $\left(\phi_{i}, \boldsymbol{\sigma}_{\eta, i}^{2}\right)$ jointly in one block marginalized over $\boldsymbol{\mu}_{i}$ and $\boldsymbol{H}$ and then in another block sample $\left(\boldsymbol{\mu}_{i}, \boldsymbol{H}\right)$ conditional on the rest in the model. To save computational cost, we do not impose the additional reweighting step introduced in Kim et al. (1998).

Given that $\boldsymbol{H}$ is drawn, we can then obtain $\boldsymbol{\epsilon}_{t}=\boldsymbol{V}_{t}^{-1 / 2} \boldsymbol{f}_{t}^{*}$ to estimate the correlations and the correlation-level parameters. Now, since the factors are observed and we have $\boldsymbol{\epsilon}_{t}$, the estimation procedure for $\boldsymbol{P}_{t}, \boldsymbol{A}, d$, and $k$ is exactly the same as that given in Asai and McAleer (2009). To sample from the complicated and non-conjugate univariate posterior distributions of $d$ and $k$, following Asai and McAleer (2009), we adopt the adaptive rejection Metropolis sampling (ARMS) of Gilks et al. (1995). The complete MCMC procedure is given as follows:

Step 0: Initialize $\boldsymbol{B}, \boldsymbol{\Omega}, \boldsymbol{s}, \boldsymbol{\omega}, \boldsymbol{H}, k, d$, and $\boldsymbol{A}$.

Step 1: Sample $\boldsymbol{B} \mid$ rest, then sample $\sigma_{j}^{2} \mid$ rest for $j=1, \ldots, p$. 
Step 2: Sample $\boldsymbol{\phi}, \boldsymbol{\sigma}_{\eta}^{2} \mid \boldsymbol{F}^{*}, \boldsymbol{s}$ and $\boldsymbol{\mu}, \boldsymbol{H} \mid \boldsymbol{F}^{*}, \boldsymbol{s}, \boldsymbol{\phi}, \boldsymbol{\sigma}_{\eta}^{2}$ using the sampler of Kim et al. (1998).

Step 3: Obtain the standardized factors $\boldsymbol{\epsilon}_{t}=\boldsymbol{V}_{t}^{-1 / 2} \boldsymbol{f}_{t}^{*}$ from the sample.

Step 4: Sample $\boldsymbol{P}_{t}$ from $\boldsymbol{P}_{t} \mid$ rest, and then obtain $\boldsymbol{\Sigma}_{\epsilon, t}=\left(\operatorname{diag} \boldsymbol{P}_{t}\right)^{-\frac{1}{2}} \boldsymbol{P}_{t}\left(\operatorname{diag} \boldsymbol{P}_{t}\right)^{-\frac{1}{2}}$ for $t=1, \ldots, T$.

Step 5: Sample $\boldsymbol{A}$ |rest.

Step 6: Sample $d \mid$ rest using ARMS.

Step 7: Sample $k \mid$ rest using ARMS.

Step 8: Go to step 1.

Looping steps 1 to 8 is a complete sweep of the MCMC sampler. It is worth noting that Steps 2 to 4 link the SV processes to the factor correlations. To deal with this part, Asai and McAleer (2009) adopt a two-stage procedure. In the first stage they estimate the SV parameters $\boldsymbol{\omega}$ and the log-volatilities $\boldsymbol{H}$ in one MCMC procedure and obtain the standardized series $\epsilon_{t i}=U_{t i} f_{t i}$ with $U_{t i}=\frac{1}{M} \sum_{l=1}^{M} \exp \left[-\frac{1}{2} h_{t i}^{(l)}\right]$, where $x^{(l)}$ denotes the $l$ th draw of the $M$ MCMC iterations. Then, in the second stage, based on the series $\boldsymbol{\epsilon}_{t}=\left(\epsilon_{t 1}, \ldots, \epsilon_{t q}\right)$, they estimate $\left\{\boldsymbol{P}_{t}\right\}$ and the correlation parameters $(\boldsymbol{A}, d, k)$ using another MCMC procedure. Clearly, the strategy does not conduct the MCMC estimation in a joint sense, which is arguably undesirable and improper in at least two respects. First, the method obtains the estimates the log-volatilities first and then plug in the estimates to run another separate MCMC. This manner averages out different samples of volatilities. Therefore, in the inference of the correlation-level parameters, we actually work with only one fixed set of log-volatilities and residuals $\epsilon$. Secondly, for the purpose of prediction, we need to sample $\boldsymbol{h}_{t+1}^{(l)}$ and then obtain $\boldsymbol{\Sigma}_{\epsilon, t}^{(l)}$, for $l=1, \ldots, M$. The plug-in method cannot be applied in this case. Unlike the two-stage scheme of Asai and McAleer (2009), our algorithm makes draws for $\boldsymbol{\omega}$ and $\boldsymbol{H}$ and then directly obtains the standardized series for the correlation parameters in each single iteration. In this way, we conduct estimation jointly with a full MCMC procedure, and the prediction can be performed directly using the usual MCMC methods. 


\section{Simulation Study}

\section{2 $\quad 3.1$ Simulated Data Example}

3 In this subsection, we use simulated data to illustrate how O-DCFMSV works. Note that the 4 illustration is based on a single run since at the very beginning we wish to present the result

5 visually. A complete simulation study based on multiple replications is provided later. We set $6 \quad p=10$ observed series and $q=2$ factors with a sample size $T=1,000$. The true data generating 7 process (DGP) is described by:

8 (i) Measurement equation:

$$
\begin{aligned}
& \boldsymbol{B}=\left(\begin{array}{rrrrrrrrrr}
1.00 & 0.30 & -0.05 & 0.99 & 0.99 & -0.10 & 0.00 & 0.56 & 0.00 & 0.00 \\
0.00 & 1.00 & 0.34 & 0.00 & 0.00 & 0.95 & 0.95 & 0.00 & 0.00 & 0.30
\end{array}\right)^{\prime} \\
& \boldsymbol{\Omega}=\operatorname{diag}(0.05,0.1,0.13,0.24,0.35,0.35,0.24,0.13,0.1,0.05) \text {. }
\end{aligned}
$$

9 (ii) SV structures:

$$
\begin{aligned}
h_{1, t+1} & =\mu_{1}+0.95\left(h_{1 t}-\mu_{1}\right)+\eta_{1 t}, \mu_{1}=-0.2 \\
h_{2, t+1} & =\mu_{2}+0.98\left(h_{2 t}-\mu_{2}\right)+\eta_{2 t}, \mu_{2}=-0.5 \\
\left(\begin{array}{l}
\eta_{1 t} \\
\eta_{2 t}
\end{array}\right) & \sim \mathrm{N}\left(\left[\begin{array}{l}
0 \\
0
\end{array}\right],\left[\begin{array}{cc}
0.1^{2} & 0 \\
0 & 0.27^{2}
\end{array}\right]\right)
\end{aligned}
$$

10

(iii) Factor correlation level:

$$
\boldsymbol{A}=\left(\begin{array}{cc}
1 & 0.05 \\
0.05 & 1
\end{array}\right)^{-1}=\left(\begin{array}{cc}
1.003 & -0.050 \\
-0.050 & 1.003
\end{array}\right), k=25, d=0.8
$$

From (iii) and the initial value $\boldsymbol{P}_{0}=\boldsymbol{I}_{q}$ we can simulate a sequence of covariance matrices $\left\{\boldsymbol{P}_{t}\right\}$, from which we can obtain the correlation matrices $\boldsymbol{\Sigma}_{\epsilon, t}$ using Eq. (44). Given $\left\{\boldsymbol{\Sigma}_{\epsilon, t}\right\}$ together with (ii), we can generate two hidden systematic factors with time-varying correlation $\rho_{t}=\left[\boldsymbol{\Sigma}_{\epsilon, t}\right]_{2,1}$. Then, given the factors we can generate ten observed series $\boldsymbol{Y}$ with the setting (i).

The MCMC study is conducted with 20,000 iterations, where the first $L=10,000$ draws are taken as burn-ins and the remaining $M=10,000$ are preserved. The program for the estimation 
is coded in OX by Doornik (2007). Table 1 summarizes the estimation results. We output the posterior means and the $95 \%$ intervals based on the $M$ draws. The posterior mean is calculated by averaging the MCMC draws. The $95 \%$ credible interval is constructed using the $(2.5 \%, 97.5 \%)$ percentiles of the simulated draws. We can see that, out of the $p q+p+3 q+q(q+1) / 2+2=41$ parameters, there is only one, $b_{72}$, not covered by the $95 \%$ credible interval.

In O-DCFMSV, one of the primary interests is in capturing the time-varying factor correlation. The factor correlation provides very useful information since it can reflect the market condition as we will see later. The top panel of Figure 1 displays the correlation fits. The smoothed estimate at $t$ is calculated by the posterior mean $\hat{\rho}_{t}=M^{-1} \sum_{l=1}^{M}\left[\boldsymbol{\Sigma}_{\epsilon, t}\right]_{21}^{(l)}$, where $\boldsymbol{\Sigma}_{\epsilon, t}^{(l)}$ is the $l$ th draw of the preserved MCMC iterations based on smoothing. Note that we draw $\boldsymbol{\Sigma}_{\epsilon, t}^{(l)}$ using Step 4 in the algorithm detailed in Section 2.3.2. The grey line is the true correlation and the black line represents the fits. We can observe that, though the fitted result appears smoother than the true values, the model in general well captures the pattern of the dynamic correlations, both the movements and the average level. To measure the performance, following Asai and McAleer (2009), we calculate two performance measures, both of which are based upon the mean absolute error (MAE) of the smoothing estimates. The first is $\operatorname{MAE}_{\rho} \equiv \frac{1}{T} \sum_{t}\left|\hat{\rho}_{t}-\rho_{t}\right|$, which measures the quality of the correlation estimates. We obtain the $\mathrm{MAE}_{\rho}=0.208$. This suggests a satisfactory result, as we can see that the correlation varies in the (wide) range $(-0.6,0.9)$.

The second measure is used for evaluating the VaR estimates. This measure is meaningful to the O-DCFMSV model since one important application of the asset-return factor model is to obtain the VaR estimates of the portfolios through factor structures. Suppose that we have a vector of portfolio asset weights $\boldsymbol{w}$. According to Barbieri et al. (2009) and Chib et al. (2006), under the assumptions of normality and a zero mean, the $5 \%$ VaR of the simulated portfolio at time $t$ is estimated by $1.645 \cdot \hat{\sigma}_{P_{t}}$, where $\hat{\sigma}_{P_{t}}$ denotes the posterior mean of the portfolio standard deviation $\sigma_{P_{t}}=\left[\boldsymbol{w}^{\prime}\left(\boldsymbol{B} \boldsymbol{V}_{t}^{1 / 2} \boldsymbol{\Sigma}_{\epsilon, t} \boldsymbol{V}_{t}^{1 / 2} \boldsymbol{B}^{\prime}+\boldsymbol{\Omega}\right) \boldsymbol{w}\right]^{\frac{1}{2}}$. (In fact this is the $95 \%$ quantile of the predictive density when the mean is zero, but by symmetry it is the negative 
1 of the $5 \%$ quantile). Suppose that our asset holdings are equally weighted, which means that

$2 \boldsymbol{w}=\frac{1}{10} \mathbf{1}$. The true and estimated VaR are shown in the bottom panel of Figure 1, where the 3 grey line represents the true $\mathrm{VaR}$ and the black line is the estimate. It is readily seen that 4 both the movement and the magnitude are nicely captured. The MAE measure is obtained by ${ }_{5} \quad \mathrm{MAE}_{\mathrm{VaR}} \equiv \frac{1}{T} \sum_{t}\left|\mathrm{VaR}_{t}^{e s t}-\mathrm{VaR}_{t}^{0}\right|$, where $\operatorname{VaR}_{t}^{\text {est }}=1.645 \cdot \hat{\sigma}_{P_{t}}$ and $\operatorname{VaR}_{t}^{0}=1.645 \cdot \sigma_{P_{t}}$. We have 6 the result $\mathrm{MAE}_{\mathrm{VaR}}=0.105$, which is quite satisfactory, for the true VaR is fluctuating in a 7 range from 0.5 to 1.5 . 
Table 1: MCMC results for the simulated data. TRUE is the true value of the parameter. MEAN is the posterior mean. (LOWER, UPPER) are the boundaries of the $95 \%$ interval.

\begin{tabular}{rrrrr}
\hline & TRUE & MEAN & LOWER & UPPER \\
\hline$B_{11}$ & 1.000 & 0.989 & 0.975 & 1.003 \\
$B_{21}$ & 0.300 & 0.303 & 0.286 & 0.321 \\
$B_{31}$ & -0.050 & -0.044 & -0.064 & -0.024 \\
$B_{41}$ & 0.990 & 0.969 & 0.946 & 0.993 \\
$B_{51}$ & 0.990 & 1.009 & 0.987 & 1.031 \\
$B_{61}$ & -0.100 & -0.080 & -0.107 & -0.053 \\
$B_{71}$ & 0.000 & 0.000 & -0.031 & 0.030 \\
$B_{81}$ & 0.560 & 0.569 & 0.532 & 0.605 \\
$B_{91}$ & 0.000 & -0.016 & -0.052 & 0.021 \\
$B_{10,1}$ & 0.000 & 0.007 & -0.037 & 0.051 \\
$B_{12}$ & 0.000 & 0.012 & -0.024 & 0.050 \\
$B_{22}$ & 1.000 & 0.995 & 0.950 & 1.041 \\
$B_{32}$ & 0.340 & 0.328 & 0.296 & 0.359 \\
$B_{42}$ & 0.000 & -0.008 & -0.047 & 0.031 \\
$B_{52}$ & 0.000 & -0.020 & -0.043 & 0.003 \\
$B_{62}$ & 0.950 & 0.937 & 0.908 & 0.965 \\
$B_{72}$ & 0.950 & 0.918 & 0.899 & 0.938 \\
$B_{82}$ & 0.000 & -0.006 & -0.030 & 0.019 \\
$B_{92}$ & 0.000 & -0.008 & -0.022 & 0.006 \\
$B_{10,2}$ & 0.300 & 0.298 & 0.281 & 0.315 \\
$\sigma_{1}^{2}$ & 0.050 & 0.052 & 0.048 & 0.057 \\
$\sigma_{2}^{2}$ & 0.100 & 0.099 & 0.091 & 0.108 \\
$\sigma_{3}^{2}$ & 0.130 & 0.124 & 0.113 & 0.135 \\
$\sigma_{4}^{2}$ & 0.240 & 0.230 & 0.211 & 0.251 \\
$\sigma_{5}^{2}$ & 0.350 & 0.333 & 0.304 & 0.362 \\
$\sigma_{6}^{2}$ & 0.350 & 0.345 & 0.316 & 0.377 \\
$\sigma_{7}^{2}$ & 0.240 & 0.253 & 0.232 & 0.276 \\
$\sigma_{8}^{2}$ & 0.130 & 0.135 & 0.124 & 0.147 \\
$\sigma_{9}^{2}$ & 0.100 & 0.101 & 0.092 & 0.110 \\
$\sigma_{10}^{2}$ & 0.050 & 0.049 & 0.045 & 0.054 \\
$\mu_{1}$ & -0.200 & -0.092 & -0.280 & 0.087 \\
$\mu_{2}$ & -0.500 & -0.859 & -1.560 & -0.141 \\
$\phi_{1}$ & 0.950 & 0.945 & 0.878 & 0.981 \\
$\phi_{2}$ & 0.980 & 0.977 & 0.960 & 0.991 \\
$\sigma_{\eta, 1}$ & 0.100 & 0.125 & 0.076 & 0.211 \\
$\sigma_{\eta, 2}$ & 0.270 & 0.222 & 0.168 & 0.285 \\
$a_{11}$ & 1.003 & 0.934 & 0.820 & 1.034 \\
$a_{12}$ & -0.050 & -0.042 & -0.080 & -0.018 \\
$a_{22}$ & 1.003 & 0.970 & 0.793 & 1.211 \\
$d$ & 0.800 & 0.756 & 0.587 & 0.856 \\
& & & & \\
\hline & 2500 & 20.797 & 10.406 & 35.057 \\
\hline
\end{tabular}



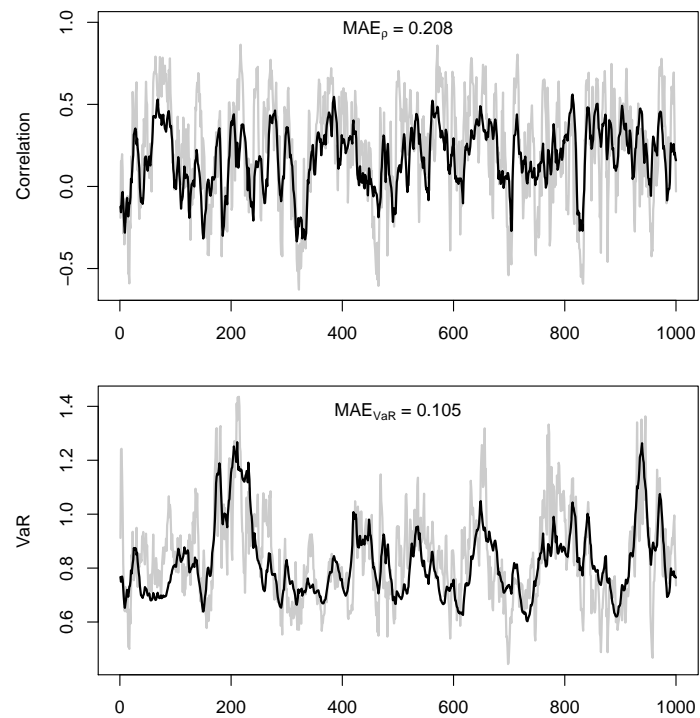

Figure 1: Factor correlations and VaR estimates. The top panel shows the true correlation process $\rho_{t}$ (grey line) and its posterior mean $\hat{\rho}_{t}$ (solid black line). The bottom panel shows $\operatorname{VaR}_{t}^{0}$ (grey line) and its posterior mean $\mathrm{VaR}_{t}^{\text {est }}$ (solid black line).

\subsection{Model Comparison}

To complete the illustration, we finish the simulation study by comparing O-DCFMSV with the benchmark, the model of Philipov and Glickman (2006b, hereafter PG), as it is also a Wishart FMSV model with dynamic factor correlations. The model specification is:

$$
\begin{aligned}
\boldsymbol{y}_{t} \mid \boldsymbol{B}, \boldsymbol{f}_{t}, \boldsymbol{\Omega} & \sim \mathrm{N}_{p}\left(\boldsymbol{B} \boldsymbol{f}_{t}, \boldsymbol{\Omega}\right), \\
\boldsymbol{f}_{t} \mid \boldsymbol{P}_{t} & \sim \mathrm{N}_{q}\left(\mathbf{0}, \boldsymbol{P}_{t}\right), \\
\boldsymbol{P}_{t}^{-1} \mid \boldsymbol{P}_{t-1}^{-1}, \boldsymbol{S}_{t-1} & \sim W_{q}\left(\boldsymbol{P}_{t-1}^{-1} \mid k, \boldsymbol{S}_{t-1}\right),
\end{aligned}
$$

2 where the matrix $\boldsymbol{P}_{t}$ is a factor covariance matrix, the meaning of the matrix $\boldsymbol{A}$ and the scalar 3 parameters $d$ and $k$ are the same as those in O-DCFMSV. Here we define the scale matrix as ${ }_{4} \boldsymbol{S}_{t}=\frac{1}{l} \boldsymbol{P}_{+}^{-\frac{d}{2}} \boldsymbol{A} \boldsymbol{P}_{t}^{-\frac{d}{2}}$, which is the form of (5) . It should be noted that, as mentioned in Section 2.1, 5 Philipov and Glickman (2006b) use the BEKK-type specification (6) for $\boldsymbol{S}_{t}$, however, in order 6 to remove the effect caused by different parameterizations, we adopt the setting (5) instead 

decomposition.

We take (i), (ii), and (iii) used in last section as the true data generation process (DGP) for O-DCFMSV. For the true DGP of PG's model, we drop (ii) and use only (i) and (iii), since the model does not assume SV structures on the factors. Two datasets with different DGPs are generated and fitted with both models. To evaluate the performance, we calculate the KullbackLeibler (KL) divergence as a measure of how far away the distribution given the estimated covariance is from that given the truth. Let $\boldsymbol{\Sigma}_{t}^{0}$ and $\hat{\boldsymbol{\Sigma}}_{t}$ be the true and estimated covariance matrices, respectively. Let $p_{t}^{0}=p\left(\boldsymbol{y}_{t} \mid \boldsymbol{\Sigma}_{t}^{0}\right)$ denote the density of $\boldsymbol{y}_{t}$ given true covariance matrix of $\boldsymbol{y}_{t}$; also, let $p_{t}^{\text {est }}=p\left(\boldsymbol{y}_{t} \mid \hat{\boldsymbol{\Sigma}}_{t}\right)$ be the density of $\boldsymbol{y}_{t}$ with the estimated covariance matrix plugged in instead of the true covariance matrix. Under normality, the KL divergence between $p_{t}^{0}$ and $p_{t}^{\text {est }}$ is

$$
\begin{aligned}
\mathrm{KL}\left(p_{t}^{0} \| p_{t}^{\mathrm{est}}\right) & =\int p_{t}^{0}(y) \log \frac{p_{t}^{0}(y)}{p_{t}^{\text {est }}(y)} d y \\
& =-\frac{p}{2}+\frac{1}{2} \operatorname{tr}\left(\hat{\boldsymbol{\Sigma}}_{t}^{-1} \boldsymbol{\Sigma}_{t}^{0}\right)-\frac{1}{2} \log \left|\boldsymbol{\Sigma}_{t}^{0}\right|+\frac{1}{2} \log \left|\hat{\boldsymbol{\Sigma}}_{t}\right|
\end{aligned}
$$

${ }_{4}$ In each replication we record the mean KL divergence (MKL), which is defined by $\frac{1}{T} \sum_{t} \mathrm{KL}\left(p_{t}^{0} \| p_{t}^{\text {est }}\right)$ as a summary of the KL divergence over every $t$.

of (6) for the competing model. Asai and McAleer (2009) point out that it is possible to use either one or the other as an alternative. The matrix power $\boldsymbol{P}_{t}^{-\frac{d}{2}}$ is calculated by the spectral

ptris

Since we have two true DGPs and two models, there are four combinations, which, by the DGP-model order, are referred as O-O, O-PG, PG-O, and PG-PG. In each combination we conduct the simulation with 40 replications and record their MKL. In each replication we calculate the differenced measure by subtracting the value of the true model from that of the wrong model, i.e. [O-PG minus O-O] and [PG-O minus PG-PG]. The differenced measure is denoted by $\triangle \mathrm{MKL}$. We report the sample mean of $\triangle \mathrm{MKL}$ from the 40 replications and the standard error as the final summarized output. Table 2 summarizes the comparison results. We can see that, for both DGPs, the differenced values of [wrong minus true] are both significantly positive, which indicate that the true models win. However, we can find that the mean $\Delta \mathrm{MKL}$ 
Table 2: Results of $\triangle \mathrm{MKL}$ for the comparison of O-DCFMSV with PG's model using simulated data. The difference is defined by the false model minus the true model. Std Err is the standard error of the mean.

\begin{tabular}{lrr}
\hline True DGP & Mean $\Delta$ MKL & Std Err \\
\hline O-DCFMSV & 0.012 & 0.002 \\
PG & 0.007 & 0.002 \\
\hline
\end{tabular}

appears to be larger in the case when the true DGP is O-DCFMSV. This has an important implication that, given both the models are misspecified, PG's model is more distant from the truth than O-DCFMSV; in other words, the KL loss is greater when PG is used and O-DCFMSV is correct, than vice versa.

\section{Empirical Study}

\subsection{Factor Correlation}

This section uses two empirical examples to illustrate the applications of the O-DCFMSV model. We use three monthly Fama-French (F-F) factors obtained from Dr. Kenneth French's data library. The factors are the market excess return (Mkt), the Small-Minus-Big factor (SMB) and the High-Minus-Low factor (HML). All the factors are rescaled to $(-1,1)$ by multiplying by 0.01. The observation period is from July 1963 to December 2005 with a total sample size of 510. Figure 2 shows the time series plot of the three rescaled F-F factors. We can see that, the behavior of the volatility is quite different among different factors. For example, during the 1980's and the 90's, the volatility is small for SMB, whereas it appears to be large for Mkt and HML. In addition, we can observe that a cluster occurs around late 1990's to early 2000's in all three factors, but the magnitude of the volatility is noticeably larger in SMB and HML than in Mkt. Accordingly, we need to allow the factor volatilities to have separate dynamics in order to reflect such facts.

Prior to the examples, we first display the time series plot of the "true" factor correlations to 

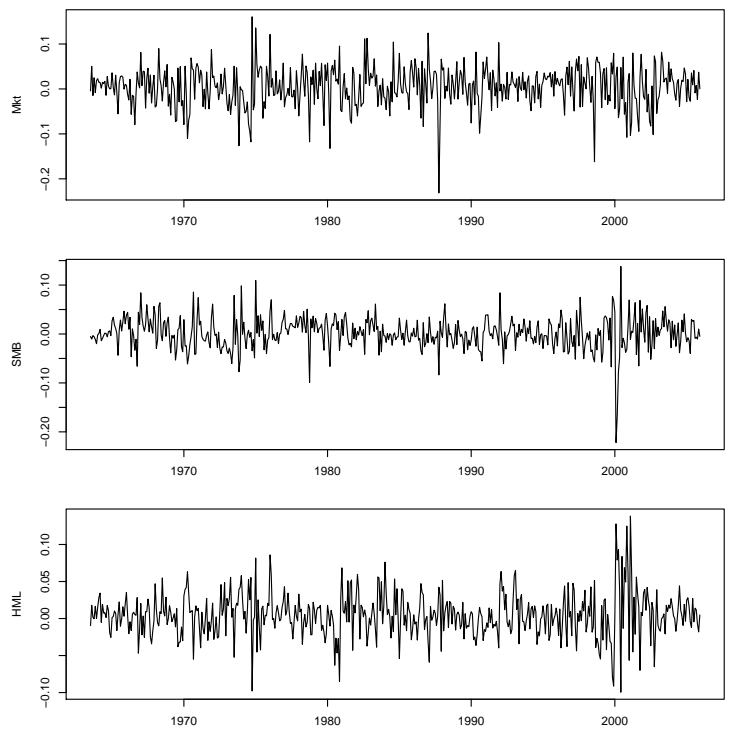

Figure 2: Time-series plot of the rescaled F-F factors, Jul 1963 - Dec 2005.

1 show that the dynamic factor correlation is a reasonable setting. The "true" correlation at time

$t$ is $\rho_{t-r}: t+r$, which we take simply as the empirical correlation calculated from the data within the window $[t-r, t+r]$. For example, if we choose $r=3$, then the correlation of (Mkt,SMB) at January 1980 is calculated by the empirical correlation of (Mkt,SMB) from October 1979 to April 1980, which approximately represents a half-year correlation. Here we choose $r=6$ for calculating the 1-year correlation, $r=12$ for the 2-year, and $r=18$, the 3-year. By rolling the windows, we obtain the "true" correlations over time.

Figure 3 shows the 1-year, 2-year, and 3-year pairwise correlations of the three factors. It is obvious that some pairs have quite large correlations during certain periods. The shaded areas account for the events having great economic impacts, which respectively are the first and second oil crisises, and the bursting of Dot-com bubble. Obviously, we can see that the factor correlations are changing over time. In particular, we can observe a common pattern: the correlations climb to a higher level or local peaks during these turbulent periods, while in the "calm" periods such as 1980's to 90's, the correlations decline to a relatively low level. This represents the well-known "correlation breakdown" phenomenon that has long been recognized 
1 in empirical data, referring to the pattern that the correlation during ordinary and stressful 2 market conditions differ substantially. See Rey (2000) for a detailed discussion. The correlation 3 breakdown implied in Figure 3] suggests that time-varying factor correlation should be considered 4 in the modeling. We would also like to point out that the main advantage of using O-DCFMSV 5 over the empirical rolling-window method to estimate factor correlations is that we can calculate 6 the credible intervals, by which we can obtain the significance relative to a specific critical level.
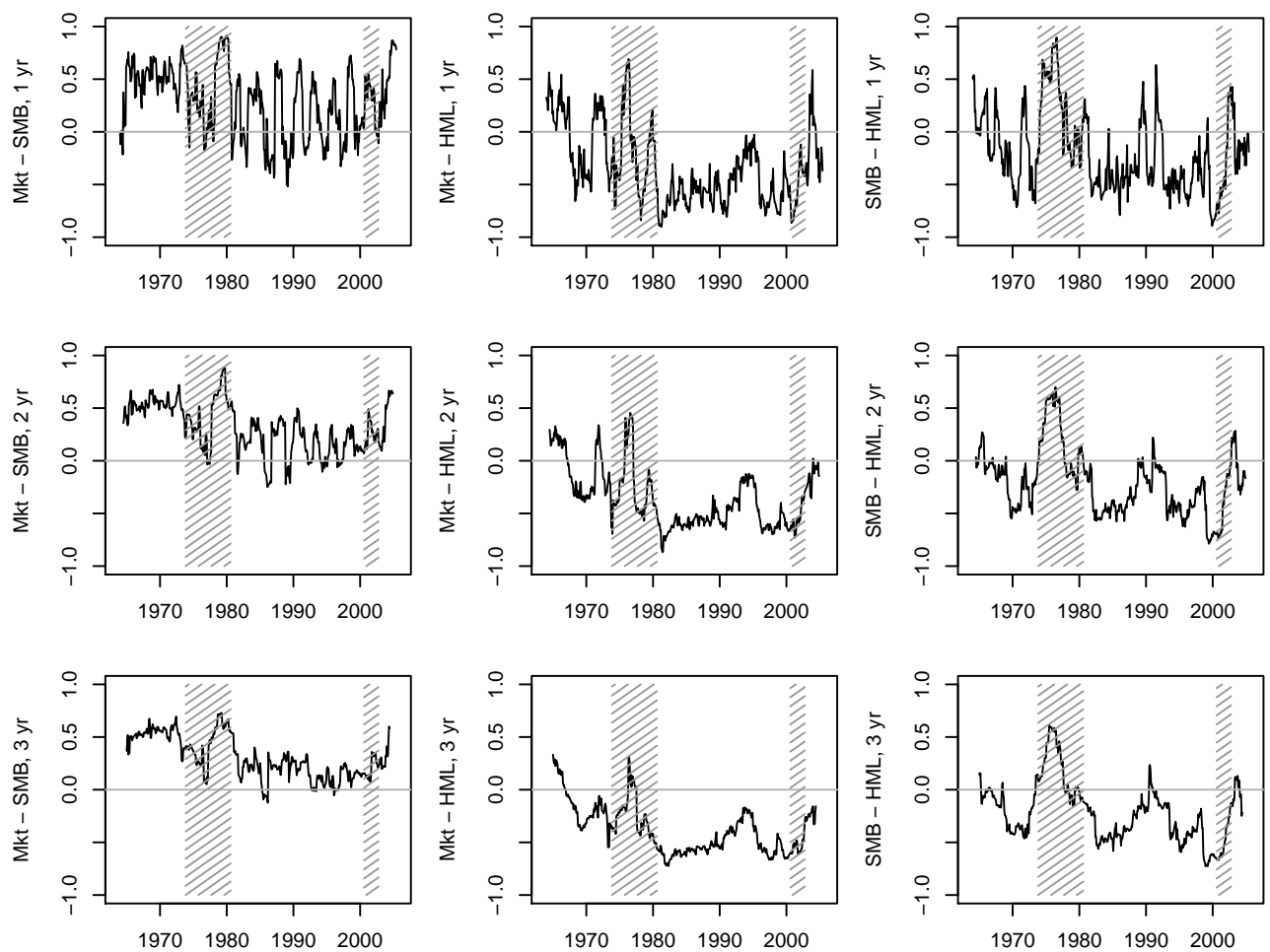

Figure 3: "True" correlations of the F-F factors Mkt-RF, SMB, and HML, Jul 1963 - Dec 2005. The shaded areas account for the events that have great economic impacts, which respectively are the first and the second oil crisis, and the Dot-com bubble burst.

\section{$7 \quad 4.2$ Example 1 - Portfolio Return Data}

8 In this first example we use the three F-F factors demonstrated in last section as the covariates.

9 The return series $\boldsymbol{Y}$ are the monthly average value weighted returns for 10 industry portfolios 
1 obtained from Dr. Kenneth French's data library. The 10 portfolios are: NoDur, Durbl, Manuf, 2 Enrgy, HiTec, Telcm, Shops, Hlth, Utils, and Other. A detailed description for these portfolios 3 can be found in the data library. Again, we first convert the data to a $(-1,1)$ scale by multiplying 4 by 0.01 . Figure 4 shows the time series plot of the data. We can observe some common clusters 5 occurring in the mid 1970's and the early 2000's, which suggests that the factor SV structure 6 can be useful. Figure 5 shows the fitted factor correlations. The black lines are the fitted values 7 and the grey lines represent the 2-year "true" factor correlations presented in Section 4.1. We 8 see that the estimates are smoother than the "true" values, but in general, the movements and 9 the magnitude of the correlations are properly captured.
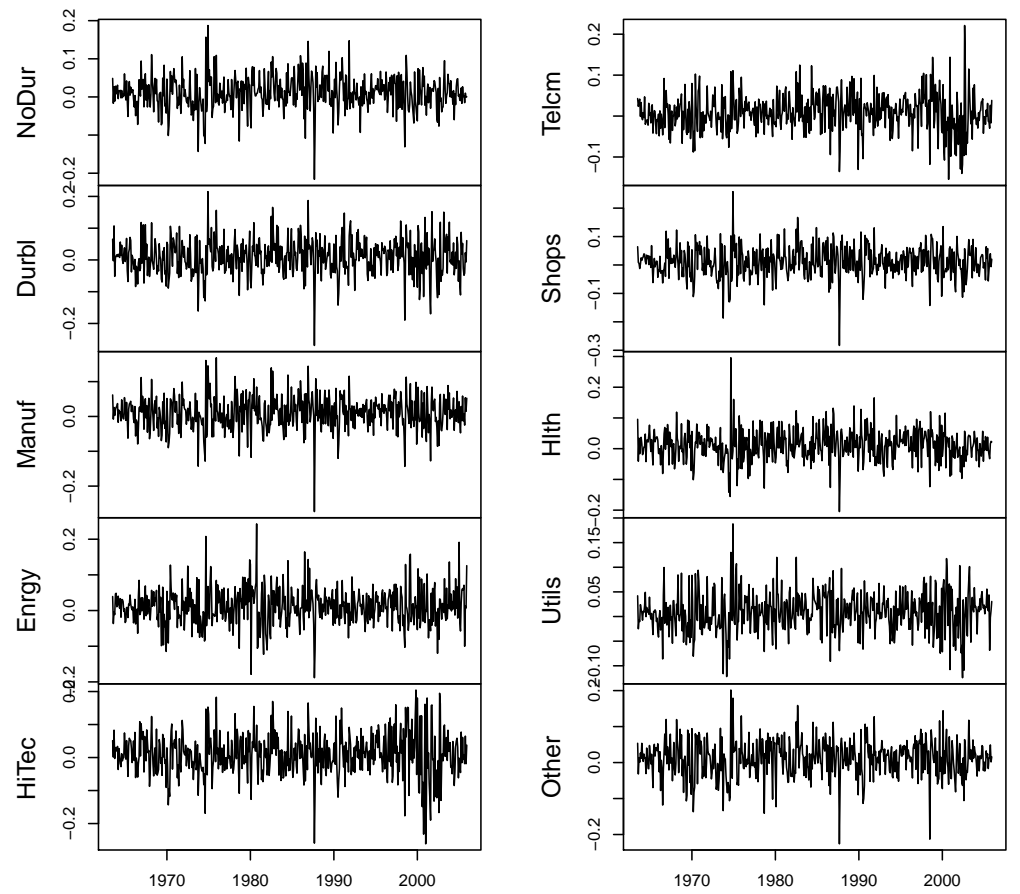

Figure 4: Time-series plot of the ten portfolios, Jul 1963 - Dec 2005. 

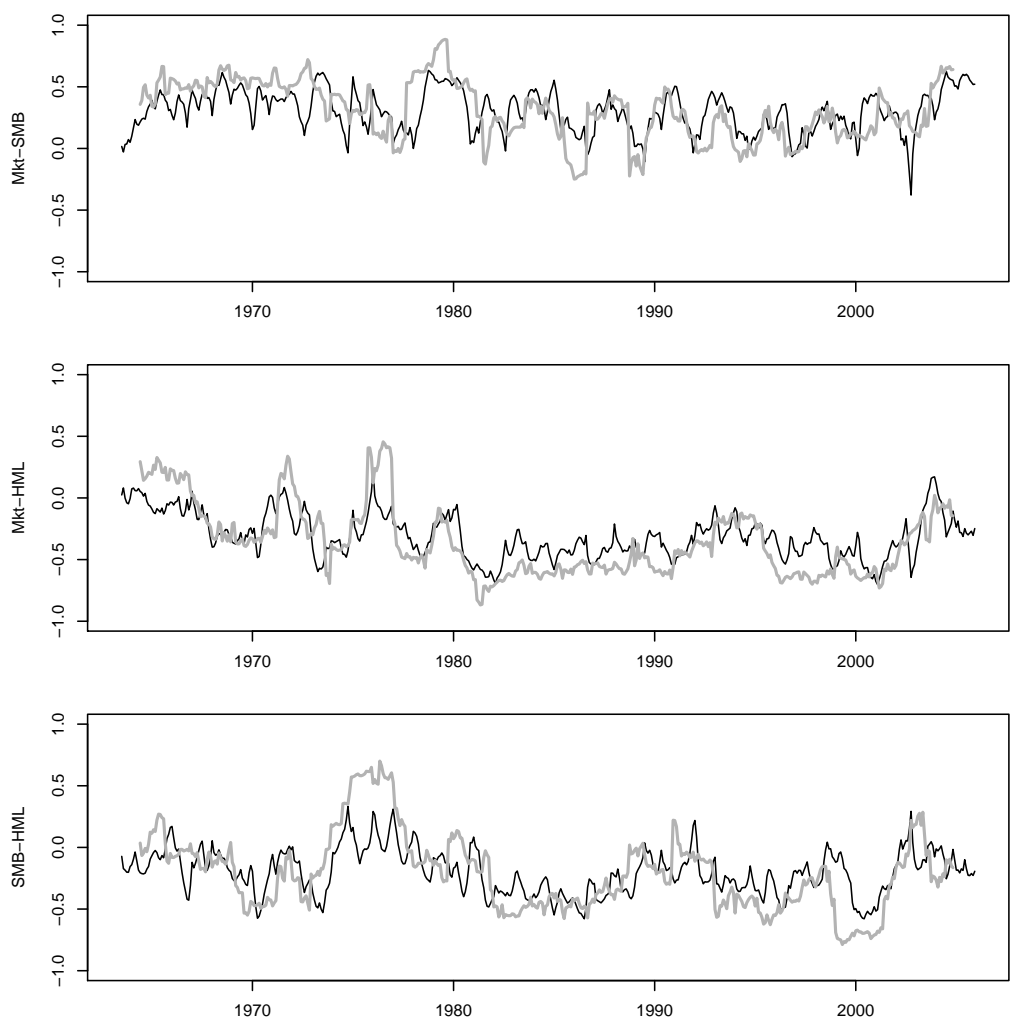

Figure 5: Estimated and "true" correlations of the factors for the portfolio data. The black lines are the fitted values and the grey lines are the "true" values.

We now compare the O-DCFMSV model with PG's model using several performance measures. The first two measures are based on the one-step-ahead predictive ability for the return covariance matrix. Notice that in this example we have 10 return series and in each month there are more than 10 transaction days. Thus, we can use daily returns to construct a nonsingular empirical covariance matrix as a proxy for the "true" covariance. Given the "true" covariance matrices, we can therefore compute $\mathrm{MAE}_{\mathrm{VaR}}$ for the equally-weighted portfolio as we do in Section 3. The empirical covariance matrix at month $t$, denoted by $\boldsymbol{\Sigma}_{t}$, is simply the sample covariance matrix constructed from the daily observations within that month with an adjustment factor of $n_{t} /\left(n_{t}-1\right)$, where $n_{t}$ is the number of transaction days within month $t$. The one-step-ahead predictor for the return covariance matrix given a model can be obtained by the 
conditional covariance:

$$
\boldsymbol{\Sigma}_{t+1}^{\mathcal{M}} \equiv \operatorname{Var}\left(\boldsymbol{y}_{t+1} \mid \mathcal{F}_{t} ; \mathcal{M}\right)=\boldsymbol{B} \operatorname{Var}\left(\boldsymbol{f}_{t+1} \mid \mathcal{F}_{t} ; \mathcal{M}\right) \boldsymbol{B}^{\prime}+\boldsymbol{\Omega}
$$

where $\mathcal{F}_{t}=\left\{\boldsymbol{y}_{1}, \ldots, \boldsymbol{y}_{t}\right\}$ is the set of observations collected up to time $t$, and $\mathcal{M}$ denotes the model, either PG or O-DCFMSV. In the implementation, for each period $t+1$ we rerun the MCMC procedure to obtain the one-step-ahead covariance matrix. Let $\boldsymbol{\Sigma}_{t+1}^{P G}$ be the one-stepahead predictive covariance matrix of $\boldsymbol{y}_{t+1}$, which is estimated by

$$
\widehat{\boldsymbol{\Sigma}}_{t+1}^{P G} \approx \frac{1}{M} \sum_{l=1}^{M}\left[\boldsymbol{B}^{(l)} \boldsymbol{P}_{t+1}^{(l)} \boldsymbol{B}^{(l)^{\prime}}+\boldsymbol{\Omega}^{(l)}\right],
$$

1 where $M$ is the number of preserved MCMC iterations and $\boldsymbol{P}_{t+1}^{(l)} \sim \boldsymbol{P}_{t+1} \mid$ rest; $\mathcal{M}_{P G}$.

For the O-DCFMSV model, the factor follows:

$$
\boldsymbol{f}_{t+1} \mid \boldsymbol{V}_{t+1}, \boldsymbol{\Sigma}_{\epsilon, t+1} \sim \mathrm{N}_{q}\left(\mathbf{0}, \boldsymbol{V}_{t+1}^{1 / 2} \boldsymbol{\Sigma}_{\epsilon, t+1} \boldsymbol{V}_{t+1}^{1 / 2}\right)
$$

Define $\boldsymbol{R}_{t+1}=\boldsymbol{V}_{t+1}^{1 / 2} \boldsymbol{\Sigma}_{\epsilon, t+1} \boldsymbol{V}_{t+1}^{1 / 2}$, where $\boldsymbol{V}_{t+1}=\operatorname{diag}\left(V_{t+1,1}, \ldots, V_{t+1, q}\right)$. From (20) , we have that

$$
\begin{aligned}
V_{t+1, i} \mid h_{t i}, \boldsymbol{\omega}_{i} & =\exp \left(h_{t+1, i}\right) \mid h_{t i}, \quad \boldsymbol{\omega}_{i} \sim \log \mathrm{N}\left(V_{t+1, i} \mid \lambda_{t i}, \sigma_{\eta, i}^{2}\right), \quad \text { where } \\
\lambda_{t i} & =\phi_{i} h_{t i}+\left(1-\phi_{i}\right) \mu_{i}, \\
V_{t+1, i}^{\frac{1}{2}} \mid h_{t i}, \boldsymbol{\omega}_{i} & \sim \log \mathrm{N}\left(V_{t+1, i}^{\frac{1}{2}} \mid \lambda_{t i} / 2, \sigma_{\eta, i}^{2} / 4\right),
\end{aligned}
$$

and $\log \mathrm{N}(x \mid a, b)$ is a $\log$ normal density in $x$ where $\log x \sim \mathrm{N}(\log x \mid a, b)$. The correlation matrix $\boldsymbol{\Sigma}_{\epsilon, t+1}$ is obtained by (44). Then, in the $l$ th MCMC iteration we can calculate $\boldsymbol{R}_{t+1}^{(l)}$. Let $\boldsymbol{\Sigma}_{t+1}^{O}$ be the one step ahead predictive variance of $\boldsymbol{y}_{t+1}$ under the O-DCFMSV model. Given $\boldsymbol{R}_{t+1}^{(l)}$, 
we have the approximation

$$
\widehat{\mathbf{\Sigma}}_{t+1}^{O} \approx \frac{1}{M} \sum_{l=1}^{M}\left(\boldsymbol{B}^{(l)} \boldsymbol{R}_{t+1}^{(l)} \boldsymbol{B}^{(l)^{\prime}}+\boldsymbol{\Omega}^{(l)}\right) .
$$

To evaluate the predictive accuracy for the $5 \%$ VaR predictions for the equally-weighted portfolio, we calculate

$$
\mathrm{MAE}_{\mathrm{VaR}} \equiv \frac{1}{N} \sum_{t}\left|\mathrm{VaR}_{t+1}^{e s t}-\mathrm{VaR}_{t+1}^{0}\right|
$$

where $N$ is the number of forecast periods and the quantity $\operatorname{VaR}_{t+1}^{0}=1.645 \cdot\left(\boldsymbol{w}^{\prime} \boldsymbol{\Sigma}_{t+1} \boldsymbol{w}\right)^{1 / 2}$ with $\boldsymbol{w}=p^{-1} \mathbf{1}$ being the weight vector. We calculate the estimate $\operatorname{VaR}_{t+1}^{e s t}$ using

$$
\operatorname{VaR}_{t+1}^{e s t}=1.645 \times\left(M^{-1} \sum_{l=1}^{M}\left[\boldsymbol{w}^{\prime} \widehat{\boldsymbol{\Sigma}}_{t+1}^{\mathcal{M}} \boldsymbol{w}\right]^{(l)}\right)^{\frac{1}{2}}
$$

In addition to the $M A E_{\mathrm{VaR}}$ measure, some authors suggest calculating the difference between the "true" and predicted covariance matrices in an elementwise sense. Following Ledoit et al. (2003), we calculate the root-mean-square error (RMSE) based on the Frobenius norm (FN):

$$
\mathrm{FN}=\frac{1}{N} \sum_{t}\left\|\boldsymbol{\Sigma}_{t+1}-\widehat{\boldsymbol{\Sigma}}_{t+1}^{\mathcal{M}}\right\|=\frac{1}{N} \sum_{t}\left[\sum_{i, j}\left(\left[\boldsymbol{\Sigma}_{t+1}\right]_{i j}-\left[\widehat{\boldsymbol{\Sigma}}_{t+1}^{\mathcal{M}}\right]_{i j}\right)^{2}\right]^{1 / 2}
$$

Because both $\mathrm{MAE}_{\mathrm{VaR}}$ and FN measure deviations from "true" values, a smaller value indicates a better model. Here we calculate the ratio of PG to O-DCFMSV for these measures so that we can compare the deviations. We output the mean values of the ratios over all the prediction periods as the summarized results.

Besides using the empirical covariance error-based measures, we also evaluate model performance in terms of the predictive quality for the return series. To do this, following Geweke and Amisano (2010), we first obtain the one-step-ahead log predictive score (LPS) and then calculate the cumulative $\log$ predictive Bayes factor. The one-step-ahead LPS evaluated at $\boldsymbol{y}_{t+1}$ under the 
specific model $\mathcal{M}$ is given by

$$
\operatorname{LPS}\left(\boldsymbol{y}_{t+1} \mid \mathcal{F}_{t} ; \mathcal{M}\right)=\log p\left(\boldsymbol{y}_{t+1} \mid \mathcal{F}_{t} ; \mathcal{M}\right)
$$

where the predictive density $p\left(\boldsymbol{y}_{t+1} \mid \mathcal{F}_{t} ; \mathcal{M}\right)$ is calculated by

$$
\begin{aligned}
p\left(\boldsymbol{y}_{t+1} \mid \mathcal{F}_{t} ; \mathcal{M}\right) & =\int p\left(\boldsymbol{y}_{t+1} \mid \mathcal{F}_{t} ; \boldsymbol{\theta}_{\mathcal{M}}\right) p\left(\boldsymbol{\theta}_{\mathcal{M}} \mid \mathcal{F}_{t}\right) d \boldsymbol{\theta}_{\mathcal{M}} \\
& \approx \frac{1}{M} \sum_{l=1}^{M} p\left(\boldsymbol{y}_{t+1} \mid \boldsymbol{x}_{t+1}^{(l)}, \boldsymbol{\theta}_{\mathcal{M}}^{(l)}\right) \\
& =\frac{1}{M} \sum_{l=1}^{M} \mathrm{~N}_{p}\left(\boldsymbol{y}_{t+1} \mid \boldsymbol{B}^{(l)} \boldsymbol{f}_{t+1}^{(l)}, \boldsymbol{\Omega}^{(l)} ; \mathcal{M}\right),
\end{aligned}
$$

where $\theta_{\mathcal{M}}$ is the set of parameters for the model $\mathcal{M}$ and $\boldsymbol{x}_{t+1}$ is the latent state vector. Then we can calculate the cumulative log predictive Bayes factor of Model 1 against Model 0, which is defined by

$$
\begin{aligned}
\log \left(B_{1,0}\right) & =\sum_{t} \log p\left(\boldsymbol{y}_{t} \mid \mathcal{F}_{t-1} ; \mathcal{M}_{1}\right)-\sum_{t} \log p\left(\boldsymbol{y}_{t} \mid \mathcal{F}_{t-1} ; \mathcal{M}_{0}\right) \\
& =\sum_{t}\left[\operatorname{LPS}\left(\boldsymbol{y}_{t} \mid \mathcal{F}_{t-1} ; \mathcal{M}_{1}\right)-\operatorname{LPS}\left(\boldsymbol{y}_{t} \mid \mathcal{F}_{t-1} ; \mathcal{M}_{0}\right)\right]
\end{aligned}
$$

In addition, we calculate the LPS for the equally-weighted portfolio $\boldsymbol{w}^{\prime} \boldsymbol{y}_{t+1}$, say, LPS-EW:

$$
\begin{aligned}
\operatorname{LPS}-\mathrm{EW}\left(\boldsymbol{w}^{\prime} \boldsymbol{y}_{t+1} \mid \mathcal{F}_{t}, \mathcal{M}\right) & =\log P\left(\boldsymbol{w}^{\prime} \boldsymbol{y}_{t+1} \mid \mathcal{F}_{t}, \mathcal{M}\right) \\
& \approx \log \left[\frac{1}{M} \sum_{l=1}^{M} \mathrm{~N}\left(\boldsymbol{w}^{\prime} \boldsymbol{y}_{t+1} \mid \boldsymbol{w}^{\prime} \boldsymbol{B}^{(l)} \boldsymbol{f}_{t+1}^{(l)}, \boldsymbol{w}^{\prime} \boldsymbol{\Omega}^{(l)} \boldsymbol{w}\right)\right]
\end{aligned}
$$

The reason to calculate LPS-EW is that if the model performs better in this measure, then we have evidence to believe that the model should also be better in forecasting the VaR for an equally-weighted portfolio. In this sense, we can regard LPS-EW as an alternative to MAE $\mathrm{VaR}_{\text {. }}$ Similar to LPS, we then calculate the cumulative log predictive Bayes factor of Model 1 against 
Model 0 for the equally-weighted portfolio:

$$
\log \left(B_{1,0}^{E W}\right)=\sum_{t}\left[\operatorname{LPS}-\mathrm{EW}\left(\boldsymbol{y}_{t} \mid \mathcal{F}_{t-1} ; \mathcal{M}_{1}\right)-\operatorname{LPS}-\mathrm{EW}\left(\boldsymbol{y}_{t} \mid \mathcal{F}_{t-1} ; \mathcal{M}_{0}\right)\right]
$$

1

The cumulative log predictive Bayes factor has a simple criterion for checking statistical significance. According to Geweke and Amisano (2010), the evaluation is conducted via the log scoring rule described in Gneiting and Raftery (2007). The detailed log scoring rule is given in Kass and Raftery (1995), of which we use the following criterion: if $\log \left(B_{1,0}\right)<0$, the evidence is in favor of Model 0 ; if $\log \left(B_{1,0}\right) \in[0,1)$, the evidence is not worth more than a bare mention; if $\log \left(B_{1,0}\right) \in[1,3)$, the evidence is positive in favor of Model 1 ; if $\log \left(B_{1,0}\right) \in[3,5)$, the evidence is strongly in favor of Model 1 ; if $\log \left(B_{1,0}\right)>5$, we have very strong evidence in favor of Model 1.

We use a three-year out-of-sample prediction period, from January 2006 to December 2008, with a total length $N=36$. This time frame covers two market conditions: before 2007, when the market is relatively calm, and afterwards when the market is relatively volatile due to the subprime crisis. Therefore, we can compare model performance across different market conditions. Here, the one-step-ahead prediction is conducted on a rolling basis, i.e., if we use observations $\boldsymbol{y}_{1}, \ldots, \boldsymbol{y}_{T}$ to forecast $\boldsymbol{y}_{T+1}$, then in next period, $\boldsymbol{y}_{T+1}$ is included as a sample for the prediction of $\boldsymbol{y}_{T+2}$. Table 3 (a) summarizes the results of the comparison using the empirical covariance error-based measures. R-MAE $\mathrm{VaR}_{\mathrm{R}}$ and R-FN denote the ratio of PG to O-DCFMSV for $\mathrm{MAE}_{\mathrm{VaR}}$ and $\mathrm{FN}$, respectively. We see that the mean ratio for $\mathrm{MAE}_{\mathrm{VaR}}$ is 1.22, suggesting that the deviance of PG is $22 \%$ larger than that of O-DCFMSV, which is a considerable difference. The mean ratio for $\mathrm{FN}$ is 1.19 , which also suggests a considerably large difference. Table 3 (b) is the summarized comparison results using cumulative log predictive Bayes factors. $\log \left(B_{O, P G}\right)$ and $\log \left(B_{O, P G}^{E W}\right)$ respectively denote the cumulative $\log$ predictive Bayes factor of O-DCFMSV against PG for $\boldsymbol{y}_{t+1}$ and $\boldsymbol{w}^{\prime} \boldsymbol{y}_{t+1}$. We see that $\log \left(B_{O, P G}\right)=14.94>$ 5 , suggesting very strong evidence in favor of O-DCFMSV. Similarly, for the equally-weighted 
Table 3: Results for the comparison of O-DCFMSV with PG's model using F-F portfolio data. R-MAE ${ }_{V a R}$ and R-FN are the ratios of PG to O-DCFMSV in MAE ${ }_{V a R}$ and FN, respectively.

(a) Empirical error-based measures.

\begin{tabular}{lc}
\hline Measure & Mean \\
\hline R-MAE $_{\text {VaR }}$ & 1.218 \\
R-FN & 1.191 \\
\hline
\end{tabular}

(b) cumulative log predictive Bayes factor.

\begin{tabular}{lr}
\hline Measure & Value \\
\hline $\log \left(B_{O, P G}\right)$ & 14.940 \\
$\log \left(B_{O, P G}^{E W}\right)$ & 13.176 \\
\hline
\end{tabular}

portfolio, we have $\log \left(B_{O, P G}^{E W}\right)=13.18>5$, which again strongly supports O-DCFMSV. In conclusion, all the evidence strongly suggests that O-DCFMSV outperforms PG's model.

The results in Table 3 (a) and (b) are aggregated over the prediction period and do not show how the two models perform at each time point. To be more convincing, we examine the "periodwise" performance. Figure 6 shows the period-by-period results for MAE $\mathrm{VaR}_{\mathrm{R}}$ and FN. It is readily seen that the values of O-DCFMSV are constantly smaller than those of PG's. Figure 7 displays the period-by-period plot of the difference in LPS and LPS-EW (O-DCFMSV minus PG). From Figure 7 we observe that the differences in LPS and LPS-EW of O-DCFMSV minus PG are constantly greater than 0, which shows that the LPS and LPS-EW of O-DCFMSV are constantly larger than those of PG's model. The only noticeable drop occurs at October 2008, which is the month right after the bankruptcy filing of Lehman Brothers and the bailout of Fannie Mae and Freddie Mac. The market was extremely volatile at that time. In fact, according to the result not shown here, the values of the predictive density functions for both models are only about $\exp (-87)$ to $\exp (-86)$, which suggests that in such an extreme environment, both models appear to be equally unlikely (i.e., they do not hold). Consequently, the result at this time point is arguably unrepresentative. Overall, based on these results, we conclude that O-DCFMSV generally performs better than the PG model in terms of one-step-ahead prediction.

\subsection{Example 2 - Stock Return Data}

The second example fits the monthly stock return data. We collect 20 historical stock prices from Yahoo! Finance. The observation period is January 1977 - June 2007, with 366 observations. We 

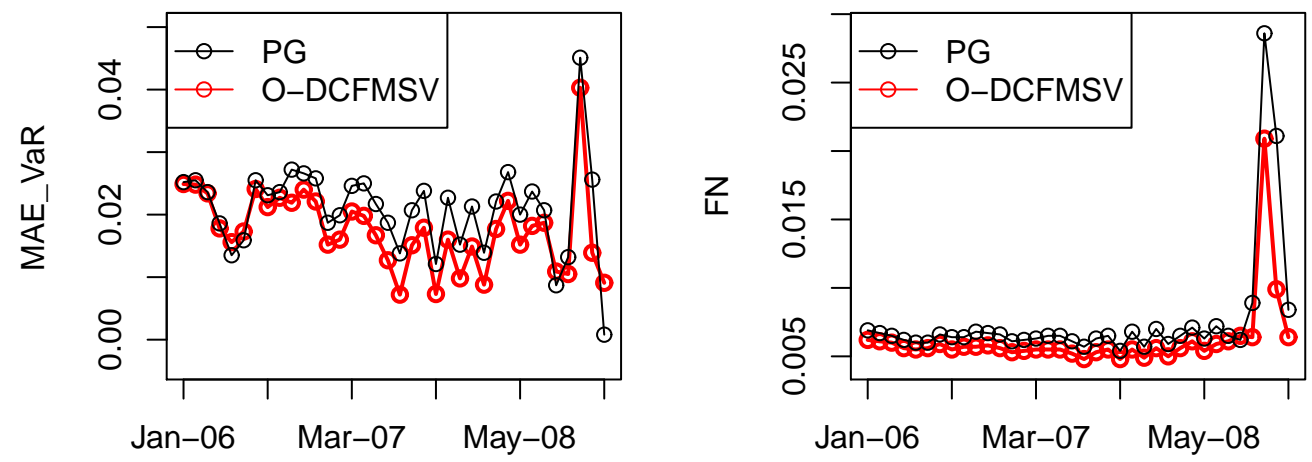

Figure 6: Period-by-period comparison using $\mathrm{MAE}_{\mathrm{VaR}}$ and FN. The portfolio return data.
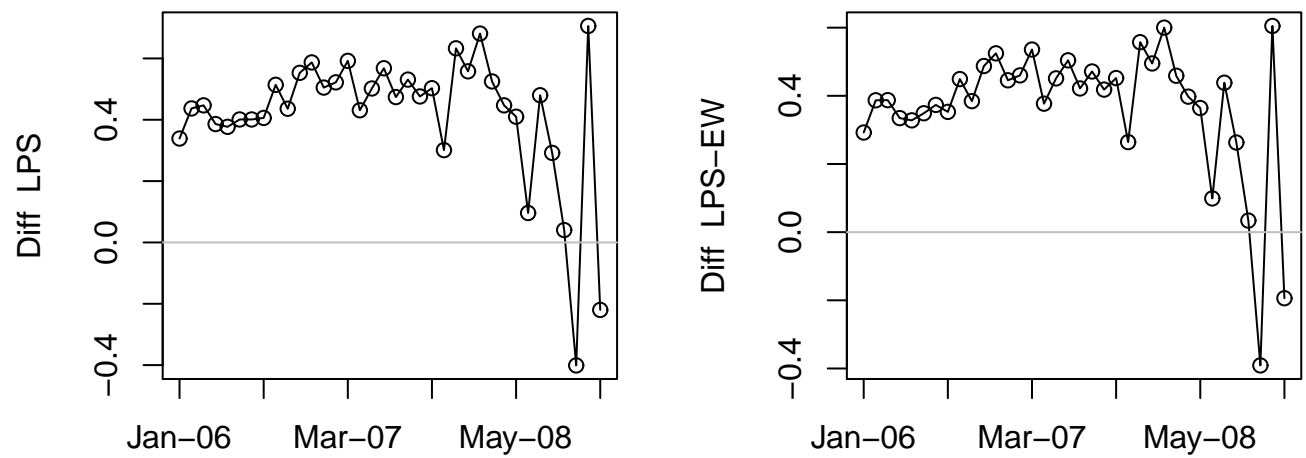

Figure 7: Difference of the predictive log-likelihood for the returns and for the equally-weighted portfolio returns. The portfolio return data.

1 calculate the stock returns by taking $\log P_{t, j}-\log P_{t-1, j}, t=2, \ldots, 366$ and $j=1, \ldots, 20$, where

$2 P_{t, j}$ is the price of the $j$ th stock at time $t$. This generates a set of return data with a sample size $3 T=365$. Similar to the example given in Philipov and Glickman (2006b), in this illustration we 4 model the stock returns using two pairs of factors, (Mkt, SMB) and (Mkt, HML), respectively.

5 We also compare O-DCFMSV to PG based on the one-step-ahead prediction quality. The out6 of-sample period is again January 2006 - December 2008. Notice that in this case we have 20 7 stock returns, but during the sample period not every month has at least 20 transaction days; 8 for this reason, unlike Example 1, here we do not calculate the empirical covariance error-based 
Table 4: Results for the comparison of O-DCFMSV with PG using stock return data.

\begin{tabular}{lrr}
\hline Measure & $($ Mkt,SMB $)$ & (Mkt,HML) \\
\hline $\log \left(B_{O, P G}\right)$ & 7.200 & 7.272 \\
$\log \left(B_{O, P G}^{E W}\right)$ & 6.408 & 6.480 \\
\hline
\end{tabular}

measures.

Table 4 shows the aggregate results, from which we can see that, no matter what pair of factors is used, the cumulative $\log$ predictive Bayes factors $\log \left(B_{O, P G}^{E W}\right)$ and $\log \left(B_{O, P G}\right)$ are both greater than 5, suggesting very strong evidence in favor of the O-DCFMSV model. Figure 8 and Figure 9 respectively display the period-by-period differences in LPS and LPS-EW (ODCFMSV minus PG) for the pairs (Mkt, SMB) and (Mkt, HML). As one can see, similar to what we observe in Example 1, the differenced values are uniformly greater than 0 in both cases except the one at October 2008. The reason is the same. At this month, no matter what pair of factors is used, the values of the predictive density functions for both models are as low as $\exp (-102)$ to $\exp (-101)$. Clearly, both models fail to work under such a market condition. Thus, again, we argue that the differences obtained at this time point may not be meaningful. Regardless of this outlier, O-DCFMSV performs uniformly better over the out-of-sample period.

Another natural question to ask is which of the two combinations of factors provides a better explanation to the data. This is a model selection question which in its generality asks how many and which factors should be used and is not discussed in Philipov and Glickman (2006b). Our solution is straightforward. We can simply compare the predictive performance of the candidate models using the cumulative log predictive Bayes factor. For instance, in this illustration we have two O-DCFMSV models, one with the factors (Mkt, SMB), denoted by MS, and the other with (Mkt, HML), MH. Table 5 summarizes the comparison result of the two models in terms of $\log \left(B_{M S, M H}\right)$ and $\log \left(B_{M S, M H}^{E W}\right)$. We see that, both of the cumulative log predictive Bayes scores show positive (but not strong) evidence in favor of (Mkt,SMB); therefore, we may conclude that, for a two-factor O-DCFMSV model, (Mkt,SMB) is a better choice for the data. Furthermore, 

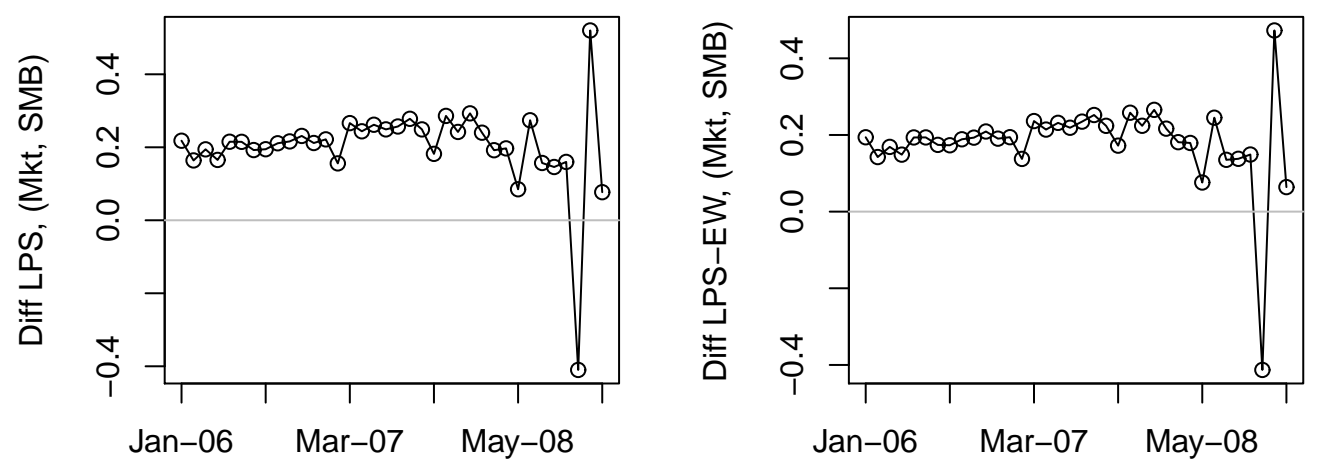

Figure 8: Difference of the predictive log-likelihood for the returns and for the equally-weighted portfolio returns. (Mkt, SMB). The stock return data.
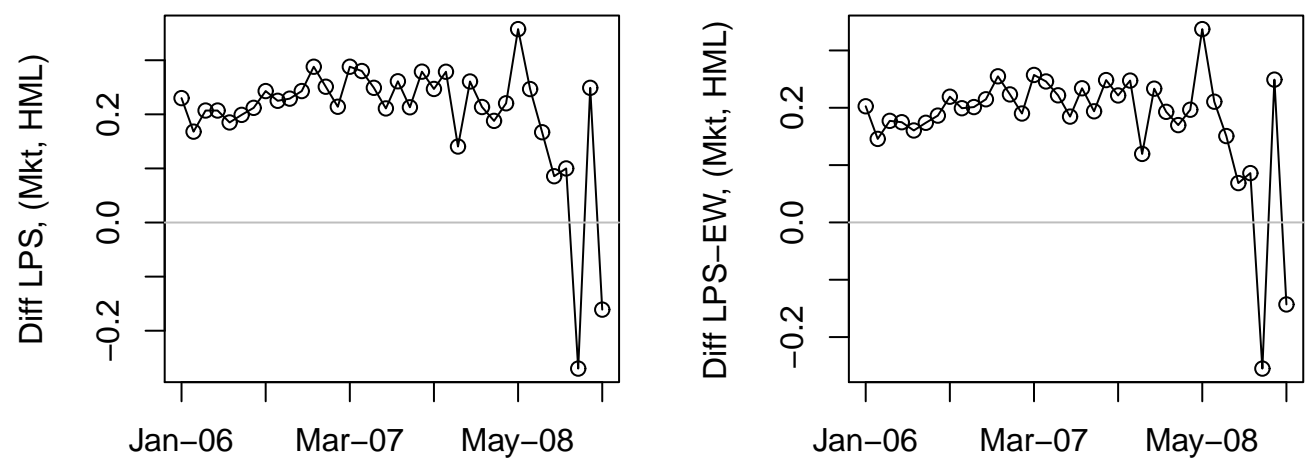

Figure 9: Difference of the predictive log-likelihood for the returns and for the equally-weighted portfolio returns. (Mkt, HML). The stock return data.

1 if we have models that contain different numbers of factors, we can also use this approach to 2 select the "best" model or the optimal number of factors.

\section{${ }_{3} 5 \mathrm{SV}$ on the Errors}

4 It is commonly seen in financial studies that daily and weekly data exhibit more volatility than 5 monthly data. For this reason, we may consider allowing each of the idiosyncratic errors to 6 follow an independent SV process, see, e.g. Pitt and Shephard (1999) and Chib et al. (2006). 
Table 5: The model comparison result using stock return data. The models are O-DCFMSV given (Mkt,SMB) and (Mkt,HML) as the covariates.

\begin{tabular}{ll}
\hline Measure & Value \\
\hline $\log \left(B_{M S, M H}\right)$ & 1.269 \\
$\log \left(B_{M S, M H}^{E W}\right)$ & 1.187 \\
\hline
\end{tabular}

1 We modify the model form (11) as

$$
\boldsymbol{y}_{t}=\boldsymbol{B} \boldsymbol{f}_{t}+\boldsymbol{e}_{t}, \quad \boldsymbol{e}_{t}=\boldsymbol{\Lambda}_{t}^{1 / 2} \boldsymbol{u}_{t}
$$

where $\boldsymbol{f}_{t}$ and $\boldsymbol{e}_{t}$ are independent and $\boldsymbol{u}_{t} \sim \mathrm{N}_{p}(\mathbf{0}, \boldsymbol{I})$. The scaling matrix $\boldsymbol{\Lambda}_{t}=\operatorname{diag}\left(e^{h_{t 1}}, \ldots, e^{h_{t p}}\right)$, where $\left\{h_{t j}, j=1, \ldots, p\right\}$ is the log-volatility of the error terms following the SV process:

$$
h_{t j}=\mu_{j}+\phi_{j}\left(h_{t j}-\mu_{j}\right)+\eta_{t j}, \quad \eta_{t j} \stackrel{i i d}{\sim} \mathrm{N}\left(0, \sigma_{\eta, j}^{2}\right) .
$$

Note that, in previous sections we use the index $i=1, \ldots, q$ for the SV processes of the factor volatilities. Here, for the log-volatilities of the errors, we use the index $j=1, \ldots, p$. With the specification (13) we need to change the sampling scheme for $\boldsymbol{B}$. Following Geweke and Zhou (1996) and Lopes and West (2004), we set the priors $\boldsymbol{b}_{j} \sim \mathrm{N}_{q}\left(\mathbf{0}, c_{0 j}^{2} \boldsymbol{I}_{q}\right)$, where $\boldsymbol{b}_{j}$ is the $j$ th row of $\boldsymbol{B}$. Throughout we choose $c_{0 j}=5$, which defines an uninformative prior for each $\boldsymbol{b}_{j}$. Let $\boldsymbol{y}_{j}$ be the $j$ th column of $\boldsymbol{Y}$ and $\boldsymbol{h}_{j}=\left\{h_{1 j}, \ldots, h_{t j}\right\}$. Given the likelihood function:

$$
\mathcal{L}\left(\boldsymbol{b}_{j} \mid \boldsymbol{y}_{j}, \boldsymbol{h}_{j}, \boldsymbol{F}\right) \propto \exp \left[-\frac{1}{2}\left(\boldsymbol{y}_{j}-\boldsymbol{F} \boldsymbol{b}_{j}\right)^{\prime} \boldsymbol{\Lambda}_{j}^{-1}\left(\boldsymbol{y}_{j}-\boldsymbol{F} \boldsymbol{b}_{j}\right)\right]
$$

where $\boldsymbol{\Lambda}_{j}=\operatorname{diag}\left(e^{h_{1 j}}, \ldots, e^{h_{t j}}\right)$. The conditional posterior for $\boldsymbol{b}_{j}$ is given by:

$$
P\left(\boldsymbol{b}_{j} \mid \text { rest }\right) \propto \exp \left\{-\frac{1}{2 \sigma_{j}^{2}}\left[\left(\boldsymbol{b}_{j}-\boldsymbol{\mu}_{b_{j}}\right)^{\prime} \boldsymbol{\Sigma}_{b_{j}}^{-1}\left(\boldsymbol{b}_{j}-\boldsymbol{\mu}_{b_{j}}\right)\right]\right\},
$$


$1 \quad$ where $\boldsymbol{\Sigma}_{b_{j}}=\left(c_{0 j}^{-2} I+\boldsymbol{F}^{\prime} \boldsymbol{\Lambda}_{j}^{-1} \boldsymbol{F}\right)^{-1}$ and $\boldsymbol{\mu}_{b_{j}}=\boldsymbol{\Sigma}_{b_{j}} \boldsymbol{F}^{\prime} \boldsymbol{\Lambda}_{j}^{-1} \boldsymbol{y}_{j}$. For $\left\{\mu_{j}, \phi_{j}, \sigma_{\eta, j}\right\}$ and $h_{t j}$, in a same manner, we use the integration sampler of Kim et al. (1998) to make draws. The sampling scheme for the other parameters remains unchanged.

To know how much we gain from adding individual SV processes on the idiosyncratic error terms, using both monthly and daily data, we compare the O-DCFMSV with SV on the errors (SV-Err) to the original model, O-DCFMSV. The model performance is compared in terms of the cumulative log predictive Bayes factors. The first comparison is based on monthly data, where we use the same dataset as in the first example of Section 4.2. Similarly, the sample period is July 1963 - December 2005 and the out-of-sample period is three-years long covering January 2006 - December 2008, with a length $N=36$. Table 6(a) summarizes the results of the comparison. We can see that for the monthly portfolio data, O-DCFMSV beats SV-Err in predicting $\boldsymbol{y}_{t+1}$ but not in the equally-weighted portfolio $\boldsymbol{w}^{\prime} \boldsymbol{y}_{t+1}$. However, if we examine Figure 10, the period-by-period prediction results, we readily see that, in general O-DCFMSV performs better than or as well as SV-Err does. Nonetheless, just as we observed in Section 4.2 , the O-DCFMSV fails to capture the movement in returns in the single extreme period, October 2008. This failure overrides those superior performances at other periods and does not reflect the overall performance of the two models. Except for this extreme case, we should agree that O-DCFMSV suffices to model the monthly data.

In the second comparison, both models are fitted to daily data. The dataset contains 30 daily stock prices collected from Yahoo Finance. The sample period is from January 3, 2006 to July 31, 2008. We calculate the stock returns as described in Example 2. This gives us the return data of a sample size $T=649$. The out-of-sample period covers one full month, August 1 - August 29, 2008, with a total length $N=21$. Table 6(b) shows the summarized result. Obviously, we see that the evidence is in favor of SV-Err since both cumulative log predictive Bayes factors are smaller than 0 . The result suggests that, for daily data the SV-Err model should be considered. 
Table 6: Comparison results for SV-Err (S) vs. O-DCFMSV (O).

(a) Monthly portfolio data.

\begin{tabular}{lr}
\hline Measure & Value \\
\hline $\log \left(B_{O, S}\right)$ & 41.976 \\
$\log \left(B_{O, S}^{E W}\right)$ & -27.864 \\
\hline
\end{tabular}

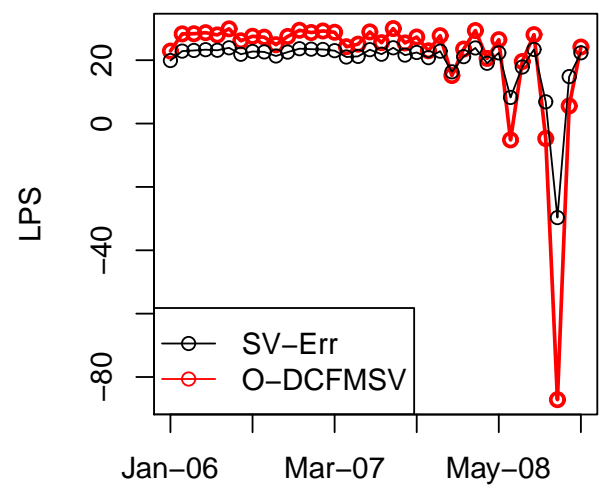

(b) Daily stock return data.

\begin{tabular}{lr}
\hline Measure & Value \\
\hline $\log \left(B_{O, S}\right)$ & -55.512 \\
$\log \left(B_{O, S}^{E W}\right)$ & -47.952 \\
\hline
\end{tabular}

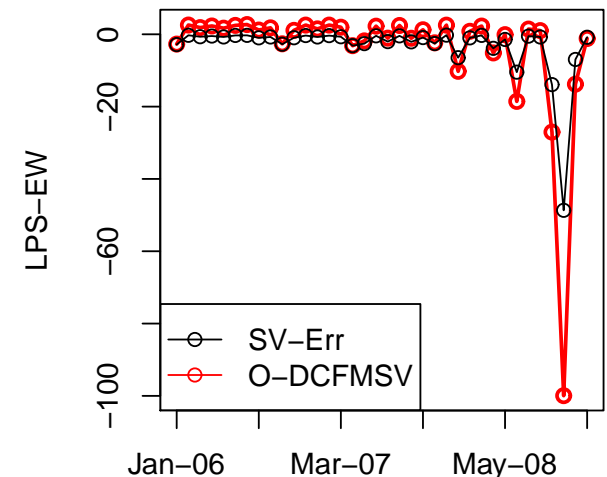

Figure 10: Comparison of SV-Err and Non-SV-Err using monthly portfolio data.

\section{Conclusion and Discussion}

2 In this paper we propose a dynamic-correlation FMSV model where the factors are observable.

3 The novelty is that we simultaneously allow the factors to have separate SV processes and the 
contain more volatility, we may need to consider the SV process on the error term. The model is easy to be extended to allow for heavy-tailedness. For example, we can add the adhoc scaled- $t$ specification suggested by Kim et al. (1998) to the idiosyncratic errors. A possible future work is to allow the factors to be latent so that the model can be more flexible. When it comes to the latent factor structure, many issues need to resolve, such as the nonidentification problem, the choice of the number of factors, and so on. We expect further research on this direction.

\section{References}

Asai, M. and McAleer, M. (2009), "The structure of dynamic correlations in multivariate stochastic volatility models," Journal of Econometric, 150, 182-192.

Barbieri, A., Chang, K., Dubikovsky, V., Fox, J., Gladkevich, A., Gold, C., and Goldberg, L. (2009), "Modeling Value at Risk with Factor," Tech. rep., MSCI Barra Research.

Chib, S., Nardari, F., and Shephard, N. (2006), "Analysis of high dimensional multivariate stochastic volatility models," Journal of Econometrics, 134, 341-371.

Doornik, J. (2007), Object-Oriented Matrix Programming Using O, Timberlake Consultants Press, London and Oxford, 3rd ed., www.doornik.com.

Geweke, J. and Amisano, G. (2010), "Comparing and evaluating Bayesian predictive distributions of asset returns," International Journal of Forecasting, 26, 216-230.

Geweke, J. and Zhou, G. (1996), "Measuring the pricing error of the arbitrage pricing theory," Review of Financial Studies, 9, 557-587.

Gilks, W., Best, N., and Tan, K. (1995), "Adaptive rejection Metropolis sampling within Gibbs sampling," Applied Statistic, 44, 455-473.

Gneiting, T. and Raftery, A. E. (2007), "Strictly Proper Scoring Rules, Prediction, and Estimation," Journal of the American Statistical Association, 102, 359-378. 
Harvey, A., Ruiz, E., and Shephard, N. (1994), "Multivariate Stochastic Variance Models," Review of Economic Studies, 61, 247-264.

Jacquier, E., Polson, N. G., and Rossi, P. E. (1995), "Models and prior distributions for multivariate stochastic volatility," Tech. Rep. 95-18, CIRANO: Scientific Series, Montreal.

Kass, R. E. and Raftery, A. E. (1995), "Bayes Factors," Journal of American Statistical Association, 90, 773-795.

Kim, S., Shephard, N., and Chib, S. (1998), "Stochastic volatility: Likelihood inference and comparison with ARCH models," Review of Economic Studies, 65, 361-393.

Ledoit, O., Santa-Clara, P., and Wolf, M. (2003), "Flexible multivariate GARCH modeling with an application to international stock markets," The Review of Economics and Statistics, 85, $735-747$.

Liesenfeld, R. and Richard, J.-F. (2006), "Classical and Bayesian Analysis of Univariate and Multivariate Stochastic Volatility Models," Econometric Reviews, 25, 335-360.

Lopes, H. and Carvalho, C. M. (2007), "Factor stochastic volatility with time varying loadings and Markov switching regimes," Journal of Statistical Planning and Inference, 137, 3082-3091.

Lopes, H. and West, M. (2004), "Bayesian model assessment in factor analysis," Statistica Sinica, 14.

Philipov, A. and Glickman, M. E. (2006a), "Multivariate stochastic volatility via Wishart processe," Journal of Business and Economic Statistics, 24, 313-328.

- (2006b), "Factor multivariate stochastic volatility via Wishart processes," Econometric Reviews, 25, 311-334.

Pitt, M. K. and Shephard, N. (1999), "Time-Varying Covariances: A Factor Stochastic Volatility Approach," in Bayesian Statistics, eds. Bernardo, J., Berger, J., Dawid, A., and Smith, A., Oxford: Oxford University Press, vol. 6, pp. 169-193. 
1 Rey, D. M. (2000), "Time-varying Stock Market Correlations and Correlation Breakdown," $2 \quad$ Financial Markets and Portfolio Management, 14, 387-412. 